\title{
ON THE EXISTENCE OF SMOOTH SOLUTIONS FOR FULLY NONLINEAR ELLIPTIC EQUATIONS WITH MEASURABLE "COEFFICIENTS" WITHOUT CONVEXITY ASSUMPTIONS*
}

\author{
NICOLAI V. KRYLOV ${ }^{\dagger}$
}

Abstract. We show that for any uniformly elliptic fully nonlinear second-order equation with bounded measurable "coefficients" and bounded "free" term one can find an approximating equation which has a unique continuous and having the second derivatives locally bounded solution in a given smooth domain with smooth boundary data. The approximating equation is constructed in such a way that it modifies the original one only for large values of the unknown function and its derivatives.

Key words. Fully nonlinear elliptic equations, Bellman's equations, finite differences.

AMS subject classifications. 35J60,39A14.

1. Introduction and main result. In this article, we consider elliptic equations

$$
H[v](x):=H\left(v(x), D v(x), D^{2} v(x), x\right)=0
$$

in subdomains of $\mathbb{R}^{d}$, where

$$
\mathbb{R}^{d}=\left\{x=\left(x_{1}, \ldots, x_{d}\right): x_{1}, \ldots, x_{d} \in \mathbb{R}=(-\infty, \infty)\right\} .
$$

Here

$$
D^{2} u=\left(D_{i j} u\right), \quad D u=\left(D_{i} u\right), \quad D_{i}=\frac{\partial}{\partial x_{i}}, \quad D_{i j}=D_{i} D_{j}
$$

We introduce $\mathcal{S}$ as the set of symmetric $d \times d$ matrices, fix a constant $\delta \in(0,1]$, and set

$$
\mathcal{S}_{\delta}=\left\{a \in \mathcal{S}: \delta|\xi|^{2} \leq a_{i j} \xi_{i} \xi_{j} \leq \delta^{-1}|\xi|^{2}, \quad \forall \xi \in \mathbb{R}^{d}\right\}
$$

where and everywhere in the article the summation convention is enforced unless specifically stated otherwise.

Recall that Lipschitz continuous functions are almost everywhere differentiable.

Assumption 1.1. (i) The function $H(u, x), u=\left(u^{\prime}, u^{\prime \prime}\right)$,

$$
u^{\prime}=\left(u_{0}^{\prime}, u_{1}^{\prime}, \ldots, u_{d}^{\prime}\right) \in \mathbb{R}^{d+1}, \quad u^{\prime \prime} \in \mathcal{S}, \quad x \in \mathbb{R}^{d},
$$

is measurable with respect to $x$ for any $u$, and Lipschitz continuous in $u$ for every $x \in \mathbb{R}^{d}$.

(ii) For any $x$, at all points of differentiability of $H(u, x)$ with respect to $u$, we have

$$
\left(H_{u_{i j}^{\prime \prime}}\right) \in \mathcal{S}_{\delta}, \quad\left|H_{u_{k}^{\prime}}\right| \leq \delta^{-1}, \quad k=1, \ldots, d, \quad 0 \leq-H_{u_{0}^{\prime}} \leq \delta^{-1}
$$

*Received April 1, 2012; accepted for publication September 5, 2012.

$\dagger 127$ Vincent Hall, University of Minnesota, Minneapolis, MN 55455, USA (krylov@math.umn. edu). The author was partially supported by NSF Grant DMS-1160569. 
(iii) Finally,

$$
\bar{H}:=\sup _{x \in \mathbb{R}^{d}}|H(0, x)|<\infty .
$$

Let $\Omega$ be an open bounded subset of $\mathbb{R}^{d}$ with $C^{2}$ boundary and take a function $g \in C^{1,1}(\bar{\Omega})$. Here is our main result, in which $K \geq 0$ is a fixed constant.

THEOREM 1.1. There is a constant $\hat{\delta} \in(0, \delta]$ depending only on $\delta$ and $d$ and there exists a function $P(u)$ (independent of $x$ ), satisfying Assumption 1.1 with $\hat{\delta}$ in place of $\delta$, such that the equation

$$
\max (H[v], P[v]-K)=0
$$

in $\Omega$ (a.e.) with boundary condition $v=g$ on $\partial \Omega$ has a unique solution $v \in C^{0,1}(\bar{\Omega}) \cap$ $C_{\text {loc }}^{1,1}(\Omega)$. In addition, for all $i, j$, and $p \in(d, \infty)$,

$$
|v|,\left|D_{i} v\right|, \rho\left|D_{i j} v\right| \leq N\left(\bar{H}+K+\|g\|_{C^{1,1}(\Omega)}\right) \quad \text { in } \quad \Omega \quad \text { (a.e.) }
$$

$$
\begin{gathered}
\|v\|_{W_{p}^{2}(\Omega)} \leq N_{p}\left(\bar{H}+K+\|g\|_{W_{p}^{2}(\Omega)}\right), \\
\|v\|_{C^{\alpha}(\Omega)} \leq N\left(\|H[0]\|_{L_{d}(\Omega)}+\|g\|_{C^{\alpha}(\Omega)}\right),
\end{gathered}
$$

where

$$
\rho(x)=\operatorname{dist}\left(x, \mathbb{R}^{d} \backslash \Omega\right),
$$

$\alpha \in(0,1)$ is a constant depending only on $d$ and $\delta, N$ is a constant depending only on $\Omega$ and $\delta$, whereas $N_{p}$ only depends on the same objects and $p$.

Finally, $P(u)$ is constructed on the sole basis of $\delta$ and $d$, it is positive homogeneous of degree one and convex in $u$.

REMARK 1.1. If we drop (1.4) and replace $C^{1,1}$ in (1.3) with $C^{1, \alpha}, \alpha \in(0,1]$, the assumptions of Theorem 1.1 about smoothness of $\Omega$ and $g$ can be somewhat relaxed. It is sufficient to have the exterior ball condition on $\Omega$ and $g \in C^{1, \alpha}(\Omega)$. Furthermore, if we multiply the derivatives in (1.3) by one more $\rho$, then one can deal with $\Omega$ such that, for each boundary point $x_{0}$ and all $r>0$ small enough with the smallness independent of $x_{0}$, there is a ball of radius $\varepsilon r$ at the distance $r$ from $x_{0}$ lying outside $\Omega$. Here $\varepsilon>0$ is a fixed constant. Of course, in that case the asserted regularity should be changed to $v \in C^{0, \beta}(\bar{\Omega}) \cap C_{l o c}^{1,1}(\Omega)$, where $\beta \in(0,1]$ is determined by other parameters of the problem. All these and other possible extensions and generalizations are left to the interested reader.

To the best of the author's knowledge Theorem 1.1 is the first uniqueness and $e x$ istence result for general fully nonlinear elliptic equations with measurable coefficients without convexity assumptions. In case $H$ is Lipschitz continuous in $x$ the theory of viscosity solutions provides the existence and uniqueness. Generally, one only knows that such solutions are in $C^{1+\alpha}$ (see Trudinger [15]). N. Nadirashvili and S. Vlădut [14] found an example in which viscosity solutions even for $H$ independent of $x$ do not have bounded second-order derivatives. 
It is also worth mentioning that M. G. Crandall, M. Kocan, and A. Święch [4] developed a theory of $L_{p}$-viscosity solutions for equations with measurable coefficients (see also the references therein).

As far as a priori estimates in Sobolev spaces are concerned, L. Caffarelli was the first author who derived interior $W_{p}^{2}$ estimates under an assumption that certain estimates hold for equations with zero "free" term, which are known to hold only for $H$ that are either convex or concave with respect to $v, D v, D^{2} v$ (see [1] and [2]). A particular case of $C^{2+\alpha}$ a priori estimates without this assumption is presented in [3]. Another case is found in [7].

The activity which started in [1] was continued by L. Wang in [16] who obtained similar interior a priori estimates for parabolic equations, by M. G. Crandall, M. Kocan, and A. Święch [4] who established the solvability in local Sobolev spaces of the boundary-value problems for fully nonlinear parabolic equations, and by N. Winter [17] who established the solvability in the global $W_{p}^{2}$-space of the associated boundaryvalue problem in the elliptic case. In the existence parts in [4] and [17] the function $H$ is supposed to be convex with respect to $D^{2} v$ and continuous in $x$ (concerning the latter assumption see [17, Remark 2.3], [9], and [4, Example 8.3]). However, in the above references the authors consider equations like (1.1) with the right-hand side which is not zero but rather a function from an $L_{p}$-space. In our setting we can only treat bounded right-hand sides.

Recently a new method, very different from the methods in the above cited references, emerged in [5] for treating fully nonlinear elliptic and parabolic equations with VMO "coefficients". Still the convexity of $H$ with respect to $D^{2} v$ is required in [5] while proving the existence result.

In our Theorem 1.1 we do not impose any convexity assumption on $H$ and allow it to be just measurable in $x$. By the way, this theorem is obviously applicable to linear equations. Yet we approximate them with nonlinear ones.

The methods of the present article are quite elementary and, apart from what is related to (1.4) and (1.5) and uniqueness, are not using anything from any existing theory of partial differential equations in the main case where $H$ depends only on pure second-order derivatives and is continuous in $x$. Our main tool is finite-difference approximations, best demonstrated in Sections 5 and 6, which the reader may like to read first.

REMARK 1.2. It is almost obvious that Assumption 1.1 (ii) is equivalent to the requirement that, for any $u \in \mathbb{R}^{d+1} \times \mathcal{S}, x, \xi \in \mathbb{R}^{d}, \eta \in\left\{ \pm e_{1}, \ldots, \pm e_{d}\right\}$, where $e_{1}, \ldots, e_{d}$ is the set of standard basis vectors in $\mathbb{R}^{d}$, and $r \geq 0$, we have

$$
\begin{gathered}
\delta|\xi|^{2} \leq H\left(u^{\prime}, u^{\prime \prime}+\xi \xi^{*}, x\right)-H\left(u^{\prime}, u^{\prime \prime}, x\right) \leq \delta^{-1}|\xi|^{2}, \\
\left|H\left(u^{\prime}+r(0, \eta), u^{\prime \prime}, x\right)-H\left(u^{\prime}, u^{\prime \prime}, x\right)\right| \leq \delta^{-1} r, \\
H\left(u^{\prime}, u^{\prime \prime}, x\right)-\delta^{-1} r \leq H\left(u^{\prime}+r(1,0), u^{\prime \prime}, x\right) \leq H\left(u^{\prime}, u^{\prime \prime}, x\right),
\end{gathered}
$$

where $(0, \eta)=\left(0, \eta_{1}, \ldots, \eta_{d}\right)$ and $(1,0)=(1,0, \ldots, 0)$.

REMARK 1.3. Estimate (1.5) follows from other assertions of Theorem 1.1 and the classical results about linear equations with measurable coefficients (see, for instance, 
Section 9.9 of [6]). Indeed, as is easy to see for $v \in W_{p}^{2}(\Omega)$ satisfying (1.2) we have that

$$
\begin{aligned}
-\max (H[0], P[0]-K) & =\max (H[v], P[v]-K)-\max (H[0], P[0]-K) \\
& =a_{i j} D_{i j} v+b_{i} D_{i} v-c v
\end{aligned}
$$

with some functions $a=\left(a_{i j}\right) \in \mathcal{S}_{\hat{\delta}},\left|b_{i}\right| \leq \hat{\delta}^{-1}, 0 \leq c \leq \hat{\delta}^{-1}$ (cf. the proof of Lemma 2.2). Furthermore, $|\max (H[0], P[0]-K)| \leq|H[0]|$.

The assertion of Theorem 1.1 concerning uniqueness in our class of functions is also a classical result derived from the Alexandrov estimate.

Here is an almost trivial generalization of Theorem 1.1 which may be useful in some applications.

THEOREM 1.2. Let $\phi \in C^{2}(\bar{\Omega})$ be a strictly positive function. Then there is a constant $\hat{\delta} \in(0, \delta]$ depending only on $\delta, \phi$, and $d$ and there exists a function $P(u)$ (independent of $x$ ), satisfying Assumption 1.1 with $\hat{\delta}$ in place of $\delta$, such that all assertions of Theorem 1.1 hold true if we replace $P[u]$ with $P[\phi u]$ and allow the constants to also depend on $\phi$.

This result is obtained from Theorem 1.1 just by replacing there $u$ and $g$ with $\phi u$ and $\phi g$, respectively.

Here is a version of Theorem 1.1 which is obtained by just replacing $H(u, x)$ with $-H(-u, x)$.

TheOREM 1.3. With $P$ from Theorem 1.1 the equation

$$
\min (H[v],-P[-v]+K)=0
$$

in $\Omega$ (a.e.) with boundary condition $v=g$ on $\partial \Omega$ has a unique solution $v \in C^{0,1}(\bar{\Omega}) \cap$ $C_{\text {loc }}^{1,1}(\Omega)$. In addition, for all $i, j$, and $p \in(d, \infty)$,

$$
\begin{gathered}
\left.|v|,\left|D_{i} v\right|, \rho\left|D_{i j} v\right| \leq N\left(\bar{H}+K+\|g\|_{C^{1,1}(\Omega)}\right) \quad \text { in } \quad \Omega \quad \text { (a.e. }\right), \\
\|v\|_{W_{p}^{2}(\Omega)} \leq N_{p}\left(\bar{H}+K+\|g\|_{W_{p}^{2}(\Omega)}\right) \\
\|v\|_{C^{\alpha}(\Omega)} \leq N\left(\|H[0]\|_{L_{d}(\Omega)}+\|g\|_{C^{\alpha}(\Omega)}\right),
\end{gathered}
$$

where $\alpha, N$, and $N_{p}$ are the constants from Theorem 1.1 .

It is an interesting issue as to what is happening to $v=v_{K}$ as $K \rightarrow \infty$, where $v_{K}$ is taken from Theorem 1.1. We have the following

Conjecture. Assume that $H(u, x)$ is Lipschitz continuous with respect to $x$ with Lipschitz constant equal to a constant times $1+|u|$. Let $w$ be a unique viscosity solution of equation (1.1) in $\Omega \in C^{3}$ with boundary condition $g \in C^{3}$. Then $\left|w-v_{K}\right| \leq$ $N / K$ where $N$ is a constant.

To conclude our comments about Theorem 1.1 we show how $P$ is constructed. By Theorems 3.1 of [10] there exists a set

$$
\left\{l_{1}, \ldots, l_{m}\right\} \subset \mathbb{Z}^{d}
$$


$m=m(\delta, d) \geq d$, chosen on the sole basis of knowing $\delta$ and $d$ and there exists a constant

$$
\hat{\delta}=\hat{\delta}(\delta, d) \in(0, \delta / 4]
$$

such that:

(i) We have

$$
e_{i}, e_{i} \pm e_{j} \in\left\{l_{1}, \ldots, l_{m}\right\}=\left\{-l_{1}, \ldots,-l_{m}\right\}
$$

for all $i, j=1, \ldots, d$ (recall that $e_{1}, \ldots, e_{d}$ is the standard orthonormal basis of $\mathbb{R}^{d}$ );

(ii) There exist real-analytic functions $\lambda_{1}(a), \ldots, \lambda_{m}(a)$ on $\mathcal{S}_{\delta / 4}$ such that for any $a \in \mathcal{S}_{\delta / 4}$

$$
a \equiv \sum_{k=1}^{m} \lambda_{k}(a) l_{k} l_{k}^{*}, \quad \hat{\delta}^{-1} \geq \lambda_{k}(a) \geq \hat{\delta}, \quad \forall k .
$$

Now introduce

$$
\mathcal{P}(z)=\max _{\substack{\hat{\delta} / 2 \leq a_{k} \leq 2 \hat{\delta}^{-1} \\ k=1, \ldots, m}} \max _{\substack{\left|b_{k}\right| \leq 2 \hat{\delta}^{-1} \\ k=1, \ldots, d_{1}}} \max _{\substack{\delta \\ k=c \leq 2 \hat{\delta}^{-1}}}\left[\sum_{k=1}^{m} a_{k} z_{k}^{\prime \prime}+\sum_{k=1}^{d} b_{k} z_{k}^{\prime}-c z_{0}^{\prime}\right],
$$

and for $u=\left(u^{\prime}, u^{\prime \prime}\right) \in \mathbb{R}^{d+1} \times \mathcal{S}$ define

$$
P\left(u^{\prime}, u^{\prime \prime}\right)=\mathcal{P}\left(u^{\prime},\left\langle u^{\prime \prime} l_{1}, l_{1}\right\rangle, \ldots,\left\langle u^{\prime \prime} l_{m}, l_{m}\right\rangle\right),
$$

where $\langle\cdot, \cdot\rangle$ is the scalar product in $\mathbb{R}^{d}$.

The rest of the article is organized as follows. In Section 2 we show that one may safely impose an additional assumption while proving Theorem 1.1. In Section 3 Theorem 1.1 is deduced from Theorem 3.2 in which even more additional assumptions are made. Then in Section 4 the function $H$ is rewritten in terms of pure second-order derivatives along certain directions.

In a quite long Section 5 we consider finite-difference approximations for equations with "constant" coefficients and prove interior estimates for the second-order differences of solutions. In Section 6 we use the results of the previous section in order to prove an analog of Theorem 1.1 for $H$, that include only pure second-order derivatives. Here the reader will see the main underlying idea of the paper, which roughly speaking is that on the set, say $\Gamma$, where the second-order derivatives of $v$ are large we have $P[v]=K$ and estimates similar to the ones from Section 5 show that the second order derivative on $\Gamma$ are controlled by their values on the boundary of $\Gamma$, where they are under control by the definition of $\Gamma$. Of course, the implementation of this idea requires first proving that there are sufficiently regular solutions of (1.2). Since we do not know how to do that, we apply the above idea at the level of finite differences.

In the final short Section 7 we prove Theorem 3.2.

In the proofs of various results in this article we use the symbol $N$ sometimes with indices to denote constants which may change from one occurrence to another and we do not always specify on which data these constants depend. In these cases the reader should remember that, if in the statement of a result there are constants called $N$ which claimed to depend only on certain parameters, then in the proof of the result the constants $N$ also depend only on the same parameters unless specifically stated otherwise. 
2. Reducing Theorem 1.1 to a particular case where $-H_{u_{0}^{\prime}} \geq \delta$. Suppose that Theorem 1.1 is true under the additional assumption that

$$
-H_{u_{0}^{\prime}} \geq \delta
$$

at all points of differentiability of $H(u, x)$ with respect to $u$. Then we are going to prove it in the original form. Take an $H$ satisfying only Assumption 1.1, take $n>0$, and consider the mapping $T_{n}: w \rightarrow v$ defined for any $w \in C(\bar{\Omega})$ and mapping it into a unique solution of

$$
\max (H[v]-v+n \chi(w / n), P[v]-K)=0
$$

in $\Omega$ (a.e.) with boundary condition $v=g$, where

$$
\chi(t)=(-1) \vee t \wedge 1
$$

By assumption $v$ is well defined and $v=T_{n} w \in C^{0,1}(\bar{\Omega}) \cap C_{l o c}^{1,1}(\Omega)$ and

$$
\begin{gathered}
\left.|v|,\left|D_{i} v\right|, \rho\left|D_{i j} v\right| \leq N\left(\bar{H}+n+K+\|g\|_{C^{1,1}(\Omega)}\right) \quad \text { in } \quad \Omega \quad \text { (a.e.) }\right) \\
\|v\|_{W_{p}^{2}(\Omega)} \leq N_{p}\left(\bar{H}+n+K+\|g\|_{W_{p}^{2}(\Omega)}\right)
\end{gathered}
$$

if $p>d$. It follows that, for each $n, T_{n}$ maps $C(\bar{\Omega})$ into its compact subset.

LEMMA 2.1. For each $n$, the mapping $T_{n}$ is continuous in $C(\bar{\Omega})$.

Proof. Let $w, w_{m} \in C(\bar{\Omega}), m=1,2, \ldots$, and assume that $\left\|w-w_{m}\right\|_{0, \Omega} \rightarrow 0$ as $m \rightarrow \infty$, where $\|\cdot\|_{0, \Omega}$ is the sup norm in $C(\bar{\Omega})$. In light of uniqueness of solutions of (2.2) with boundary condition $v=g$, to prove the lemma, it suffices to show that, at least along a subsequence, $\left\|T_{n} w-v_{m}\right\|_{0, \Omega} \rightarrow 0$, where $v_{m}=T_{n} w_{m}$. Since $T_{n} C(\bar{\Omega})$ is a compact set, there is a subsequence and a $v \in C(\bar{\Omega})$ such that $\left\|v-v_{m}\right\|_{0, \Omega} \rightarrow 0$ and $v=g$ on $\partial \Omega$. Without losing generality we may assume that the above convergence holds along the original sequence. Now we need only show that $v=T_{n} w$.

Observe that for $m \geq r$ we have

$$
\max \left(H\left[v_{m}\right]-v_{m}+n \sup _{k \geq r} \chi\left(w_{k} / n\right), P\left[v_{m}\right]-K\right) \geq 0
$$

in $\Omega$ (a.e.). Since the norms $\left\|v_{m}\right\|_{W_{d}^{2}(\Omega)}$ are bounded, by Theorems 3.5.9 and 3.5.15 of [8], whose conditions are easily checked on the basis of Remark 1.2, we have (a.e.)

$$
\max \left(H[v]-v+n \sup _{k \geq r} \chi\left(w_{k} / n\right), P[v]-K\right) \geq 0 .
$$

By letting $r \rightarrow \infty$ we get (a.e.)

$$
\max (H[v]-v+n \chi(w / n), P[v]-K) \geq 0 .
$$

One obtains the opposite inequality starting with

$$
\max \left(H\left[v_{m}\right]-v_{m}+n \inf _{k \geq r} \chi\left(w_{k} / n\right), P\left[v_{m}\right]-K\right) \leq 0 .
$$

It follows that $v=T_{n} w$ indeed and the lemma is proved. 
Now by Tikhonov's theorem we conclude that, for each $n$, there exists $v^{n} \in C(\bar{\Omega})$ such that $v^{n}=T_{n} v^{n}$. By assumption $v^{n} \in C^{0,1}(\bar{\Omega}) \cap C_{l o c}^{1,1}(\Omega)$ and

$$
\begin{gathered}
\left|D_{i} v^{n}\right|, \rho\left|D_{i j} v^{n}\right| \leq N\left(\bar{H}+\left\|v^{n}\right\|_{0, \Omega}+K+\|g\|_{C^{1,1}(\Omega)}\right) \quad \text { in } \quad \Omega \quad \text { (a.e.), } \\
\left\|v^{n}\right\|_{W_{p}^{2}(\Omega)} \leq N_{p}\left(\bar{H}+\left\|v^{n}\right\|_{0, \Omega}+K+\|g\|_{W_{p}^{2}(\Omega)}\right) .
\end{gathered}
$$

LEMMA 2.2. There is a constant $N$ depending only on the diameter of $\Omega$ and $\delta$ such that

$$
\left\|v^{n}\right\|_{0, \Omega} \leq N\left(\bar{H}+K+\|g\|_{C(\Omega)}\right) .
$$

Proof. Introduce

$$
H_{K}^{n}(u, x)=\max \left(H(u, x)-u_{0}^{\prime}+n \chi\left(u_{0}^{\prime} / n\right), P(u)-K\right)
$$

and observe that $H_{K u_{0}^{\prime}}^{n} \leq 0$ and by Hadamard's formula

$$
\begin{aligned}
H_{K}^{n}\left(u^{\prime}, u^{\prime \prime}, x\right) & -H_{K}^{n}(0, x)=u_{i j}^{\prime \prime} \int_{0}^{1} H_{K u_{i j}^{\prime \prime}}^{n}\left(t u^{\prime}, t u^{\prime \prime}, x\right) d t \\
& +\sum_{i \geq 1} u_{i}^{\prime} \int_{0}^{1} H_{K u_{i}^{\prime}}^{n}\left(t u^{\prime}, t u^{\prime \prime}, x\right) d t+u_{0}^{\prime} \int_{0}^{1} H_{K u_{0}^{\prime}}^{n}\left(t u^{\prime}, t u^{\prime \prime}, x\right) d t
\end{aligned}
$$

provided that $H^{n}(u, x)$ is differentiable with respect to $u$ at $(t u, x)$ for almost all $t \in[0,1]$. Since this happens to be the case for almost all $u$, we see that, for each $n$, there exist $\mathcal{S}_{\delta}$-valued function $a$ and real-valued functions $b_{1}, \ldots, b_{d}, c$, and $f$ satisfying $\left|b_{i}\right| \leq \delta^{-1}, c \geq 0,|f| \leq \bar{H}+K$ such that in $\Omega$ (a.e.)

$$
a_{i j} D_{i j} v^{n}+b_{i} D_{i} v^{n}-c v^{n}=f .
$$

Now our result follows by the Alexandrov maximum principle (see, for instance, Section 3.3 of [8]). The lemma is proved.

Due to this lemma one can drop $\left\|v^{n}\right\|_{0, \Omega}$ in the right-hand sides of estimates (2.3). After that it only remains to observe that for $n \geq\left\|v^{n}\right\|_{0, \Omega}$, the function $v^{n}$ satisfies (1.2) since $\chi\left(v^{n} / n\right)=v^{n} / n$ and Theorem 1.1 holds in its original form.

Hence, in the rest of the article we suppose that (2.1) holds at all points of differentiability of $H$ with respect to $u$.

\section{Further reductions of Theorem 1.1.}

1. First, we show that we may additionally assume that for any $x, y \in \mathbb{R}^{d}$ and $u=\left(u^{\prime}, u^{\prime \prime}\right)$

$$
|H(u, x)-H(u, y)| \leq N|x-y|(1+|u|),
$$

where $N$ is independent of $x, y, u$.

Indeed, if Theorem 1.1 is true in this particular case, take a nonnegative $\zeta \in$ $C_{0}^{\infty}\left(\mathbb{R}^{d}\right)$, which integrates to one, set $\zeta^{n}(x)=n^{d} \zeta(n x)$, and introduce $H^{n}(u, x)$ as the convolution of $H(u, x)$ and $\zeta^{n}$ performed with respect to $x$. Observe that $H^{n}$ satisfies (2.1) and Assumption 1.1 with the same constant $\delta$, whereas

$$
\left|H^{n}(u, x)-H^{n}(u, y)\right| \leq n|x-y| \sup _{z}|H(u, z)| \sup |D \zeta|
$$


and (3.1) is satisfied since $|H(u, z)| \leq|H(0, z)|+N(d) \delta^{-1}|u|$. Then assuming that the assertions of Theorem 1.1 are true under our additional assumption, we conclude that there exist solutions $v^{n} \in C^{0,1}(\bar{\Omega}) \cap C_{l o c}^{1,1}(\Omega)$ of

$$
\max \left(H^{n}\left[v^{n}\right], P\left[v^{n}\right]-K\right)=0
$$

in $\Omega$ (a.e.) with boundary condition $v^{n}=g$, for which estimates (1.3) and (1.4) hold with $v^{n}$ in place of $v$ with the constants $N$ and $N_{p}$ from Theorem 1.1 and with

$$
\overline{H^{n}}=\sup _{x \in \mathbb{R}^{d}}\left|H^{n}(0, x)\right| \quad(\leq \bar{H})
$$

in place of $\bar{H}$. In particular,

$$
\check{H}_{K}^{n}\left[v^{m}\right] \geq 0
$$

in $\Omega$ (a.e.) for all $m \geq n$, where

$$
\check{H}_{K}^{n}(u, x):=\sup _{k \geq n} \max \left(H^{k}(u, x), P(u)-K\right) .
$$

Furthermore, being uniformly bounded and uniformly continuous, the sequence $\left\{v^{n}\right\}$ has a subsequence uniformly converging to a function $v$, for which (1.3) and (1.4), of course, hold and $v \in C^{0,1}(\bar{\Omega}) \cap C_{l o c}^{1,1}(\Omega)$. In light of (3.3) and the fact that the norms $\left\|v^{n}\right\|_{W_{p}^{2}(\Omega)}$ are bounded, by Theorems 3.5.9 and 3.5.15 of [8] (the applicability of which is shown by an argument similar to the one in Remark 1.3) we have

$$
\check{H}_{K}^{n}[v] \geq 0
$$

in $\Omega$ (a.e.).

Then we notice that by the Lebesgue differentiation theorem for any $u$

$$
\lim _{n \rightarrow \infty} \check{H}_{K}^{n}(u, x)=\max (H(u, x), P(u)-K)
$$

for almost all $x$. Since $\check{H}_{K}^{n}(u, x)$ are Lipschitz continuous in $u$ with a constant independent of $x$ and $n$, there exists a subset of $\Omega$ of full measure such that (3.5) holds on this subset for all $u$.

We conclude that in $\Omega$ (a.e.)

$$
\max (H[v], P[v]-K) \geq 0
$$

The opposite inequality is obtained by considering

$$
\inf _{k \geq n} \max \left(H^{k}(u, x), P(u)-K\right) .
$$

2. Next, we show that one may assume that $H$ is boundedly inhomogeneous with respect to $u$. Introduce

$$
P_{0}(u)=\max _{a \in \mathcal{S}_{\delta / 2}} \max _{\substack{\left|b_{i}\right| \leq 2 \delta^{-1} \\ i=1, \ldots, d}} \max _{c \in\left[\delta / 2,2 \delta^{-1}\right]}\left(a_{i j} u_{i j}^{\prime \prime}+b_{i} u_{i}^{\prime}-c u_{0}^{\prime}\right),
$$

where the summations are performed before the maximum is taken. It is easy to see that $P_{0}[u]$ is a kind of Pucci's operator:

$$
P_{0}(u)=-(\delta / 2) \sum_{k=1}^{d} \lambda_{k}^{-}\left(u^{\prime \prime}\right)+2 \delta^{-1} \sum_{k=1}^{d} \lambda_{k}^{+}\left(u^{\prime \prime}\right)+2 \delta^{-1} \sum_{k=1}^{d}\left|u_{k}^{\prime}\right|-(\delta / 2)\left(u_{0}^{\prime}\right)^{+}+2 \delta^{-1}\left(u_{0}^{\prime}\right)^{-},
$$


where $\lambda_{1}\left(u^{\prime \prime}\right), \ldots, \lambda_{d}\left(u^{\prime \prime}\right)$ are the eigenvalues of $u^{\prime \prime}$ and $a^{ \pm}=(1 / 2)(|a| \pm a)$.

Recall that the function $P$ is introduced in the end of Section 1 and observe that

$$
P(u)=\max _{\substack{\hat{\delta} / 2 \leq a_{k} \leq 2 \hat{\delta}^{-1} \\ k=1, \ldots, m}} \max _{\substack{\left|b_{i}\right| \leq 2 \hat{\delta}^{-1} \\ i=1, \ldots, d}} \max _{\hat{\delta} / 2 \leq c \leq 2 \hat{\delta}^{-1}}\left[\sum_{i, j=1}^{d} \sum_{k=1}^{m} a_{k} l_{k i} l_{k j} u_{i j}^{\prime \prime}+\sum_{i=1}^{d} b_{i} u_{i}^{\prime}-c u_{0}^{\prime}\right] .
$$

Moreover, owing to property (ii) in the end of Section 1, the collection of matrices

$$
\sum_{k=1}^{m} a_{k} l_{k} l_{k}^{*}
$$

such that $\hat{\delta} \leq a_{k} \leq \hat{\delta}^{-1}, k=1, \ldots, m$, covers $\mathcal{S}_{\delta / 4}$. By combining this with the fact that $\hat{\delta} \leq \delta / 2$ (actually, $\hat{\delta} \leq \delta / 4$, which will be used much later) we see that

$$
\begin{aligned}
P(u) \geq & -(\delta / 4) \sum_{k=1}^{d} \lambda_{k}^{-}\left(u^{\prime \prime}\right)+4 \delta^{-1} \sum_{k=1}^{d} \lambda_{k}^{+}\left(u^{\prime \prime}\right) \\
& +4 \delta^{-1} \sum_{k=1}^{d}\left|u_{k}^{\prime}\right|-(\delta / 4)\left(u_{0}^{\prime}\right)^{+}+4 \delta^{-1}\left(u_{0}^{\prime}\right)^{-} \\
\geq & P_{0}(u)+(\delta / 4) \sum_{k=1}^{d}\left|\lambda_{k}\left(u^{\prime \prime}\right)\right|+(\delta / 4) \sum_{k=0}^{d}\left|u_{k}^{\prime}\right| .
\end{aligned}
$$

In particular, $P_{0} \leq P$ and therefore,

$$
\max (H, P-K)=\max \left(H_{K}, P-K\right),
$$

where $H_{K}=\max \left(H, P_{0}-K\right)$. It is easy to see that the function $H_{K}$ satisfies Assumption 1.1 and (2.1) with $\delta / 2$ in place of $\delta$. It also satisfies (3.1) with the same constant $N$.

Furthermore, we have the following.

LEMma 3.1. There is a constant $\kappa>0$ depending only on $\delta$ and $d$ such that for all $x \in \Omega$ and $u=\left(u^{\prime}, u^{\prime \prime}\right)$

$$
\begin{gathered}
H \leq P_{0}-\kappa\left(\sum_{i, j}\left|u_{i j}^{\prime \prime}\right|+\sum_{i}\left|u_{i}^{\prime}\right|\right)+H(0, x), \\
H_{K} \leq P-\kappa\left(\sum_{i, j}\left|u_{i j}^{\prime \prime}\right|+\sum_{i}\left|u_{i}^{\prime}\right|\right)+H^{+}(0, x) .
\end{gathered}
$$

Furthermore,

$$
\begin{gathered}
H(u, x) \leq N\left(\sum_{i, j}\left|u_{i j}^{\prime \prime}\right|+\sum_{i}\left|u_{i}^{\prime}\right|\right)+H(0, x), \\
|H(u, x)| \leq N\left(\sum_{i, j}\left|u_{i j}^{\prime \prime}\right|+\sum_{i}\left|u_{i}^{\prime}\right|\right)+|H(0, x)|,
\end{gathered}
$$

where the constant $N$ depends only on $\delta$. 
Proof. Observe that if a number $p \in(a, b), a<b$, and $y \in \mathbb{R}$, then

$$
\text { yp } \leq y^{+} b-y^{-} a \text {. }
$$

Then from Hadamard's formula

$$
\begin{aligned}
H\left(u^{\prime}, u^{\prime \prime}, x\right) & -H(0,0, x)=u_{i j}^{\prime \prime} \int_{0}^{1} H_{u_{i j}^{\prime \prime}}\left(t u^{\prime}, t u^{\prime \prime}, x\right) d t \\
& +\sum_{i \geq 1} u_{i}^{\prime} \int_{0}^{1} H_{u_{i}^{\prime}}\left(t u^{\prime}, t u^{\prime \prime}, x\right) d t+u_{0}^{\prime} \int_{0}^{1} H_{u_{0}^{\prime}}\left(t u^{\prime}, t u^{\prime \prime}, x\right) d t
\end{aligned}
$$

we obtain (see our comments regarding (2.4))

$$
\begin{aligned}
H\left(u^{\prime}, u^{\prime \prime}, x\right)-H(0,0, x) \leq & \delta^{-1} \sum_{k} \lambda_{k}^{+}\left(u^{\prime \prime}\right)-\delta \sum_{k} \lambda_{k}^{-}\left(u^{\prime \prime}\right)+\delta^{-1} \sum_{i \geq 1}\left|u_{i}^{\prime}\right| \\
& -\delta\left(u_{0}^{\prime}\right)^{+}+\delta^{-1}\left(u_{0}^{\prime}\right)^{-} \\
= & P_{0}\left(u^{\prime}, u^{\prime \prime}\right)-\delta^{-1} \sum_{k} \lambda_{k}^{+}\left(u^{\prime \prime}\right)-(\delta / 2) \sum_{k} \lambda_{k}^{-}\left(u^{\prime \prime}\right) \\
& -\delta^{-1} \sum_{i \geq 1}\left|u_{k}^{\prime}\right|-\delta^{-1}\left(u_{0}^{\prime}\right)^{-}-(\delta / 2)\left(u_{0}^{\prime}\right)^{+}
\end{aligned}
$$

and (3.8) follows since

$$
\begin{aligned}
{\left[\sum_{k}\left(\lambda_{k}^{+}\left(u^{\prime \prime}\right)+\lambda_{k}^{-}\left(u^{\prime \prime}\right)\right)\right]^{2} } & =\left(\sum_{k}\left|\lambda_{k}\left(u^{\prime \prime}\right)\right|\right)^{2} \\
& \geq \sum_{k}\left|\lambda_{k}\left(u^{\prime \prime}\right)\right|^{2}=\sum_{i, j}\left|u_{i j}^{\prime \prime}\right|^{2} \geq d^{-2}\left(\sum_{i, j}\left|u_{i j}^{\prime \prime}\right|\right)^{2} .
\end{aligned}
$$

Estimate (3.9) follows from (3.8) and (3.7). Finally, the second assertion of the lemma follows directly from the above Hadamard's formula. The lemma is proved.

In addition, $H_{K}$ is boundedly inhomogeneous with respect to $u$ in the sense that at all points of differentiability of $H_{K}(u, x)$ with respect to $u$

$$
\left|H_{K}(u, x)-H_{K u_{i j}^{\prime \prime}}(u, x) u_{i j}^{\prime \prime}-H_{K u_{r}^{\prime}}(u, x) u_{r}^{\prime}\right| \leq N\left(\left|H_{K}(0, x)\right|+K\right),
$$

where $N$ depends only on $\delta$ and $d$.

Indeed, if

$$
\kappa\left(\sum_{i, j}\left|u_{i j}^{\prime \prime}\right|+\sum_{i}\left|u_{i}^{\prime}\right|\right) \geq H^{+}(0, x)+K
$$

then by Lemma 3.1

$$
H(u, x) \leq P_{0}(u)-\kappa\left(\sum_{i, j}\left|u_{i j}^{\prime \prime}\right|+\sum_{i}\left|u_{i}^{\prime}\right|\right)+H^{+}(0, x) \leq P_{0}(u)-K,
$$

so that $H_{K}(u, x)=P_{0}(u)-K$ and the left-hand side of (3.10) is just $K$ owing to the fact that $P_{0}$ is positive homogeneous of degree one. On the other hand, if the opposite inequality holds in (3.11), then again in light of Lemma 3.1 the left-hand side of (3.10) is dominated by

$$
N\left(\sum_{i, j}\left|u_{i j}^{\prime \prime}\right|+\sum_{i}\left|u_{i}^{\prime}\right|\right)+\left|H_{K}(0, x)\right| \leq N\left(\left|H_{K}(0, x)\right|+H^{+}(0, x)+K\right),
$$


where

$$
H(0, x) \leq \max (H(0, x),-K)=H_{K}(0, x), \quad H^{+}(0, x) \leq\left|H_{K}(0, x)\right| .
$$

Furthermore, as we have noticed above $H_{K}$ satisfies Assumption 1.1 and (2.1) (with $\delta / 2$ in place of $\delta$ ) and as is easy to see $\left|H_{K}[0]\right| \leq|H[0]|+K$, which shows that in the rest of the article we may (and will) assume that not only Assumption 1.1 and (2.1) are satisfied with $\delta / 2$ in place of $\delta$ and (3.1) holds with a constant $N$, but also at all points of differentiability of $H$ with respect to $u$

$$
\left|H(u, x)-H_{u_{i j}^{\prime \prime}}(u, x) u_{i j}^{\prime \prime}-H_{u_{r}^{\prime}}(u, x) u_{r}^{\prime}\right| \leq N_{0},
$$

where $N_{0}$ is a constant and

$$
H \leq P-\kappa\left(\sum_{i, j}\left|u_{i j}^{\prime \prime}\right|+\sum_{i}\left|u_{i}^{\prime}\right|\right)+|H(0, \cdot)|,
$$

where $\kappa$ is the constant from Lemma 3.1. By the way we keep track of the value of $\delta$ in Assumption 1.1 and (2.1) because $P(u)$ is already fixed and defined by $d$ and $\delta$.

3. Finally, we show that we may assume that,

$$
H(u, x)=\operatorname{tr} u^{\prime \prime}-u_{0}^{\prime} \text { for all } u \text { if } x \text { is in a neighborhood of } \partial \Omega,
$$

that is, for an $\varepsilon>0$, we have $H(u, x)=\operatorname{tr} u^{\prime \prime}-u_{0}^{\prime}$ if $\rho(x) \leq \varepsilon$. Indeed, take a continuous function $\zeta(t), t \geq 0$ such that $\zeta(t)=1$ for $t \in[0,1], \zeta(t)=0$ for $t \geq 2$, and $0 \leq \zeta \leq 1$. Introduce

$$
H^{1 / \varepsilon}(u, x)=(1-\zeta(\rho(x) / \varepsilon)) H(u, x)+\zeta(\rho(x) / \varepsilon)\left(\operatorname{tr} u^{\prime \prime}-u_{0}^{\prime}\right) .
$$

Notice that $H^{1 / \varepsilon}$ satisfies Assumption 1.1 and (2.1) with $\delta / 2$ in place of $\delta$, satisfies (3.1) with a constant $N$ depending on $\varepsilon$ but independent of $x, y, u$, and satisfies (3.12) with the same constant $N_{0}$. As long as (3.13) is concerned, observe that by Lemma 3.1 applied to $H=\operatorname{tr} u^{\prime \prime}-u_{0}^{\prime}$ and by the inequality $P_{0} \leq P$ we have

$$
\operatorname{tr} u^{\prime \prime}-u_{0}^{\prime} \leq P-\kappa\left(\sum_{i, j}\left|u_{i j}^{\prime \prime}\right|+\sum_{i}\left|u_{i}^{\prime}\right|\right) .
$$

Then owing to (3.13)

$$
\begin{aligned}
H^{1 / \varepsilon} & \leq P-\kappa\left(\sum_{i, j}\left|u_{i j}^{\prime \prime}\right|+\sum_{i}\left|u_{i}^{\prime}\right|\right)+|(1-\zeta(\rho / \varepsilon)) H(0, \cdot)| \\
& =P-\kappa\left(\sum_{i, j}\left|u_{i j}^{\prime \prime}\right|+\sum_{i}\left|u_{i}^{\prime}\right|\right)+\left|H^{1 / \varepsilon}(0, \cdot)\right| .
\end{aligned}
$$

Therefore, if the assertions of Theorem 1.1 hold under the above additional assumptions, then we have a sequence of functions $v^{n} \in C^{0,1}(\bar{\Omega}) \cap C_{\text {loc }}^{1,1}(\Omega)$ satisfying (3.2) (with new $H^{n}=H^{1 / \varepsilon}$ for $\varepsilon=1 / n$ ).

After that by repeating literally the argument in no. 1 we come to (3.4) and since, obviously, $H^{1 / \varepsilon}(u, x) \rightarrow H(u, x)$ as $\varepsilon \downarrow 0$ for any $x \in \Omega$, we conclude that equation (3.6) holds (a.e.) and we finish the argument as in no. $\mathbf{1 .}$

As a result of the above arguments we see that to prove Theorem 1.1 it suffices to prove the following. 
ThEOREM 3.2. Suppose that Assumption 1.1 is satisfied with $\delta / 2$ in place of $\delta$. Also assume that (3.13) and (3.14) hold. Finally, assume that estimate (3.1) holds for any $x, y \in \mathbb{R}^{d}$ and $u=\left(u^{\prime}, u^{\prime \prime}\right)$ with a constant $N$ and (2.1) and (3.12) hold at all points of differentiability of $H(u, x)$ with respect to $u$.

Then the assertions of Theorem 1.1 hold true with $P$ introduced in the end of Section 1.

REMARK 3.1. One may wonder why we need (3.12) with a constant which does not enter the assertions of Theorem 3.2 in any way. The only reason to reduce general $H$ to boundedly inhomogeneous ones is that for those we can rewrite $H[u]$ in such a way that only pure second-order derivatives of $u(t, x)$ with respect to $x$ enter. Then the whole operator $\max (H[u], P[u]-K)$ also has this form.

Another possible question is: Why don't we start with $\max (H, P-K)$, which is already boundedly inhomogeneous by the above? The point is that our way to transform boundedly inhomogeneous operators does not preserve the particular structure of $\max (H, P-K)$.

4. Writing $H$ in Theorem 3.2 in a special form. Here we suppose that the assumptions of Theorem 3.2 are satisfied and take the objects introduced in the end of Section 1. Owing to the the assumptions of Theorem 3.2 by Theorem 7.1 of [10] (see the beginning of its proof in [10]) there exists a function $\mathcal{H}(z, x)$ defined for

$$
z=\left(z^{\prime}, z^{\prime \prime}\right), \quad z^{\prime}=\left(z_{0}^{\prime}, \ldots, z_{d}^{\prime}\right) \in \mathbb{R}^{d+1}, \quad z^{\prime \prime} \in \mathbb{R}^{m}, \quad x \in \mathbb{R}^{d}
$$

such that:

(i) The function $\mathcal{H}$ is Lipschitz continuous in $z$ with Lipschitz constant $\hat{\delta}^{-1}$ and there exists a constant $N^{\prime}$ such that

$$
|\mathcal{H}(z, x)-\mathcal{H}(z, y)| \leq N^{\prime}|x-y|(1+|z|)
$$

for all $x, y \in \mathbb{R}^{d}$ and $z$.

(ii) We have $\mathcal{H}(z, x)=H(u, x)$ if $z^{\prime}=u^{\prime}$ and for all $j=1, \ldots, m$

$$
z_{j}^{\prime \prime}=\left\langle u^{\prime \prime} l_{j}, l_{j}\right\rangle
$$

In particular, $\mathcal{H}(0, x)=H(0, x)$ and if $v(x)$ is a real-valued function which is twice differentiable at a point $x \in \mathbb{R}^{d}$, at this point we have

$$
H[v](x)=\mathcal{H}[v](x)
$$

where

$$
\mathcal{H}[v](x)=\mathcal{H}\left(v(x), D v(x), D_{l_{1}}^{2} v(x), \ldots, D_{l_{m}}^{2} v(x), x\right), \quad D_{l}^{2} v=v_{x_{i} x_{j}} l_{i} l_{j}
$$

(iii) At all points $(z, x)$ at which $\mathcal{H}(z, x)$ is differentiable with respect to $z$ we have

$$
\begin{gathered}
\left|\mathcal{H}_{z_{i}^{\prime}}(z, x)\right| \leq 4 \delta^{-1}, \quad i=1, \ldots, d, \\
\delta / 4 \leq-\mathcal{H}_{z_{0}^{\prime}}(z, x) \leq 4 \delta^{-1}, \quad \hat{\delta}^{-1} \geq \mathcal{H}_{z_{j}^{\prime \prime}}(z, x) \geq \hat{\delta}, \quad j=1, \ldots, m .
\end{gathered}
$$


The proofs in [10] use the fact that (3.12) holds and yield the function $\mathcal{H}$ such that, in addition, at all points $(z, x)$ at which $\mathcal{H}(z, x)$ is differentiable with respect to $z$ we also have

$$
\left|\mathcal{H}(z, x)-\left\langle z, D_{z} \mathcal{H}(z, x)\right\rangle\right| \leq 2 N_{0} .
$$

However, the latter property of $\mathcal{H}$ will not be used in the future, so that we only used assumption (3.12) to be sure that $\mathcal{H}$ with the properties (i)-(iii) exists.

5. An auxiliary equation. Some notation in this section are different from the previous ones. Fix an $h \in(0,1]$ and for $\xi \in \mathbb{R}^{d}$ and any function $\phi$ on $\mathbb{R}^{d}$ introduce

$$
T_{\xi} \phi(x)=\phi(x+h \xi), \quad \delta_{\xi}=h^{-1}\left(T_{\xi}-1\right), \quad \Delta_{\xi}=h^{-2}\left(T_{\xi}-2+T_{-\xi}\right) .
$$

Notice that $h$ enters the definition of $T_{\xi}$ and $\delta_{\xi}$ and $\Delta_{\xi}$ are usual approximations for the first and second-order derivatives along $\xi$.

Let $m \geq 1$ be an integer and let $\ell_{-m}, \ldots, \ell_{-1}, \ell_{1}, \ldots, \ell_{m}$ be some fixed vectors in $\mathbb{R}^{d}$ such that

$$
\ell_{-k}=-\ell_{k}
$$

Next denote $\Lambda=\left\{\ell_{k}: k= \pm 1, \ldots, \pm m\right\}$,

$$
\Lambda_{1}=\Lambda, \quad \Lambda_{n+1}=\Lambda_{n}+\Lambda, \quad n \geq 1, \quad \Lambda_{\infty}=\bigcup_{n} \Lambda_{n} .
$$

Let $m^{\prime} \geq 0$ be an integer $\leq m$ and let $A=\{\alpha=(a, b, c)\}$ be a closed bounded set in $\mathbb{R}^{2 m} \times \mathbb{R}^{m^{\prime}} \times \mathbb{R}$, so that

$$
\begin{gathered}
a=\left(a_{-m}, a_{-m+1}, \ldots, a_{-1}, a_{1}, \ldots, a_{m}\right) \in \mathbb{R}^{2 m}, \\
b=\left(b_{1}, \ldots, b_{m^{\prime}}\right) \in \mathbb{R}^{m^{\prime}},
\end{gathered}
$$

and $c \in \mathbb{R}$. Also let $f(\alpha, x)$ be a real-valued function defined for $\alpha \in A, x \in \mathbb{R}^{d}$.

Fix an $r \in\{1, \ldots, m\}$ and for $k= \pm 1, \ldots, \pm m$ set

$$
\delta_{h, k}=\delta_{k}=\delta_{\ell_{k}}, \quad \Delta_{h, k}=\Delta_{k}=\Delta_{\ell_{k}} .
$$

Assumption 5.1. There are constants $\delta>0$ and $K_{1}, K_{2} \in[0, \infty)$ such that

(i) For any $(a, b, c) \in A$ and all $k$ we have

$$
a_{k}=a_{-k}, \quad \delta \leq a_{k} \leq \delta^{-1}, \quad\left|b_{k}\right| \leq \delta^{-1}, \quad h b_{k}^{-} \leq a_{k}, \quad c \geq 0 ;
$$
$\mathbb{R}^{d}$

(ii) The function $f$ is continuous in $\alpha$ for any $x$ and $\left|\delta_{r} f\right| \leq K_{1}, \Delta_{r} f \geq-K_{2}$ on

For $u=\left(u^{\prime}, u^{\prime \prime}\right)$ with

$$
u^{\prime}=\left(u_{0}^{\prime}, u_{1}^{\prime}, \ldots, u_{m^{\prime}}^{\prime}\right), \quad u^{\prime \prime}=\left(u_{-m}^{\prime \prime}, \ldots, u_{-1}^{\prime \prime}, u_{1}^{\prime \prime}, \ldots, u_{m}^{\prime \prime}\right),
$$

introduce

$$
\mathcal{P}(u, x)=\max _{\alpha=(a, b, c) \in A}\left(\sum_{|k|=1}^{m} a_{k} u_{k}^{\prime \prime}+\sum_{k=1}^{m^{\prime}} b_{k} u_{k}^{\prime}-c u_{0}^{\prime}+f(\alpha, x)\right) .
$$


For any function $u$ on $\mathbb{R}^{d}$ define

$$
\mathcal{P}[u](x)=\mathcal{P}\left(u(x), \delta u(x), \delta^{2} u(x), x\right),
$$

where

$$
\begin{gathered}
\delta u=\left(\delta_{1} u, \ldots, \delta_{m^{\prime}} u\right), \\
\delta^{2} u=\left(\Delta_{-m} u, \ldots, \Delta_{-1} u, \Delta_{1} u, \ldots, \Delta_{m} u\right) .
\end{gathered}
$$

In connection with this notation a natural question arises as to why use $\ell_{k}$ along with $\ell_{-k}=-\ell_{k}$ since $\Delta_{k}=\Delta_{-k}$ and

$$
a_{k} \Delta_{k}=2 \sum_{k \geq 1} a_{k} \Delta_{k}
$$

owing to the assumption that $a_{k}=a_{-k}$. This is done for the sake of convenience of computations. For instance,

$$
\Delta_{k}(u v)=u \Delta_{k} v+v \Delta_{k} u+\left(\delta_{k} u\right)\left(\delta_{k} v\right)+\left(\delta_{-k} u\right)\left(\delta_{-k} v\right)
$$

(no summation in $k$ ). At the same time

$$
a_{k} \Delta_{k}(u v)=u a_{k} \Delta_{k} v+v a_{k} \Delta_{k} u+2 a_{k}\left(\delta_{k} u\right)\left(\delta_{k} v\right)
$$

as if we were dealing with usual partial derivatives.

Fix a nonempty finite set $Q^{o} \subset h \Lambda_{\infty}$ and let

$$
Q:=Q^{o} \cup\left\{x+h \Lambda: x \in Q^{o}\right\} .
$$

Next take a function $\eta \in C^{\infty}\left(\mathbb{R}^{d}\right)$ with bounded derivatives, such that $|\eta| \leq 1$ and set $\zeta=\eta^{2}$,

$$
\begin{gathered}
\left|\eta^{\prime}(x)\right|=\left|\eta^{\prime}(x)\right|_{h}=\sup _{k}\left|\delta_{k} \eta(x)\right|, \quad\left|\eta^{\prime \prime}(x)\right|=\left|\eta^{\prime \prime}(x)\right|_{h}=\sup _{k}\left|\Delta_{k} \eta(x)\right|, \\
\left\|\eta^{\prime}\right\|=\left\|\eta^{\prime}\right\|_{h}=\sup _{h \Lambda_{\infty}}\left|\eta^{\prime}\right|_{h}, \quad\left\|\eta^{\prime \prime}\right\|=\left\|\eta^{\prime \prime}\right\|_{h}=\sup _{h \Lambda_{\infty}}\left|\eta^{\prime \prime}\right|_{h} .
\end{gathered}
$$

Finally, let $u$ be a function on $\mathbb{R}^{d}$ such that in $Q^{o}$

$$
\mathcal{P}[u]=0
$$

and $\mathcal{P}[u] \leq 0$ on $Q \backslash Q^{o}$.

THEOREM 5.1. There exist constants $N=N(m, \delta) \geq 1$ and $N^{*}=N^{*}(m, \delta)$ such that for any constant $\nu$ satisfying

$$
\nu \geq N^{*}\left\|\eta^{\prime}\right\|+N\left(\left\|\eta^{\prime \prime}\right\|+\left\|\eta^{\prime}\right\|^{2}\right)
$$

we have in $Q$ that (recall that $a^{ \pm}=(1 / 2)(|a| \pm a)$ )

$$
\zeta^{2}\left[\left(\Delta_{r} u\right)^{-}\right]^{2} \leq \max _{Q \backslash Q^{o}} \zeta^{2}\left[\left(\Delta_{r} u\right)^{-}\right]^{2}+\left(N \nu+N^{*}\right) \bar{W}_{r}+N \nu^{-2} K_{2}^{2}+\nu^{-1} K_{1}^{2},
$$


where

$$
\bar{W}_{r}=\max _{Q}\left(\left|\delta_{r} u\right|^{2}+\left|\delta_{-r} u\right|^{2}\right)
$$

Furthermore, $N^{*}=0$ if $b \equiv 0$.

REMARK 5.1. Theorem 5.1 looks very much like Theorem 1.1 of [11]. However, in the latter the boundary of $Q^{\circ}$ is "twice fatter" and all mixed second-order differences are present under the maximum sign in the corresponding counterpart of (5.2). Our idea is to apply Theorem 5.1 to regions where at least one of pure second-order differences is large. Then outside the region all of them will be under control. Yet this does not provide any control of mixed differences on the boundary of the region and makes it impossible to apply Theorem 1.1 of [11], where the driving goal was to obtain estimates for equations with variable coefficients and estimating all mixed second-order finite differences was necessary.

In the following arguments no summation with respect to $r$ is done. The number $r$ is fixed in the beginning of the section. For simplicity of notation set

$$
u_{r r}=\Delta_{r} u, \quad u_{r}=\delta_{r} u, \quad u_{k r}=-\delta_{-k} \delta_{r} u .
$$

Notice that in the above line the last notation when $k=r$ is consistent with the first one.

In the following two lemmas the fact that $u$ is a solution of (5.1) is not used and

$$
u_{r r}^{-}=\left(u_{r r}\right)^{-} .
$$

Lemma 5.2. There exists $N=N(m, \delta)$ and $N^{*}=N^{*}(m, \delta)$ such that, if $N^{*} h \leq$ 1 , on $Q^{\circ}$ for any $\alpha=(a, b, c) \in A$ we have

$$
\begin{aligned}
-2 \zeta^{2} u_{r r}^{-}\left[a_{k} \Delta_{k}+b_{k} \delta_{k}\right] u_{r r}^{-} \geq & -\left[a_{k} \Delta_{k}+b_{k} \delta_{k}\right]\left(\zeta^{2}\left(u_{r r}^{-}\right)^{2}\right) \\
-N^{*}\left|\eta^{\prime}\right| \zeta a_{k} u_{k r}^{2}- & N\left(\left|\eta^{\prime \prime}\right|+\left|\eta^{\prime}\right|^{2}\right) \zeta\left(u_{r r}^{-}\right)^{2}-\left(N^{*}\left|\eta^{\prime}\right|^{2}+N\left|\eta^{\prime}\right|^{4}\right) \bar{W}_{r}, \\
-2 \zeta u_{r}\left[a_{k} \Delta_{k}+b_{k} \delta_{k}\right] u_{r} \geq & -\left[a_{k} \Delta_{k}+b_{k} \delta_{k}\right]\left(\zeta u_{r}^{2}\right) \\
& +\zeta a_{k} u_{k r}^{2}-N\left(\left|\eta^{\prime \prime}\right|+\left|\eta^{\prime}\right|^{2}\right) \bar{W}_{r}-N^{*}\left|\eta^{\prime}\right| \bar{W}_{r} .
\end{aligned}
$$

Furthermore, $N^{*}=0$ if $b \equiv 0$.

Proof. Here is the result of simple computations, which can be found, for instance, in the proof of Lemma 5.1 of [11]. For any $\alpha \in A$ we have

$$
\begin{aligned}
a_{k} \Delta_{k}\left(\zeta^{2}\left(u_{r r}^{-}\right)^{2}\right)= & 2 \zeta^{2} u_{r r}^{-} a_{k} \Delta_{k} u_{r r}^{-}+2 a_{k}\left[\delta_{k}\left(\zeta u_{r r}^{-}\right)\right]^{2}+4 a_{k} u_{r r}^{-}\left(\delta_{k} \zeta\right) \delta_{k}\left(\zeta u_{r r}^{-}\right) \\
& +2 a_{k}\left(u_{r r}^{-}\right)^{2}\left[\zeta \Delta_{k} \zeta-2\left(\delta_{k} \zeta\right)^{2}\right]-4 h u_{r r}^{-} a_{k}\left(\delta_{k} \zeta\right)^{2} \delta_{k} u_{r r}^{-} .
\end{aligned}
$$

We also know from Lemma 4.3 of [11] that

$$
\left|\Delta_{k} \zeta\right| \leq 2\left(\left|\eta^{\prime \prime}\right|+\left|\eta^{\prime}\right|^{2}\right), \quad\left(\delta_{k} \zeta\right)^{2} \leq N\left|\eta^{\prime}\right|^{2} \zeta+N h^{2}\left|\eta^{\prime}\right|^{4} \leq N\left|\eta^{\prime}\right|^{2} .
$$

It follows that, for any $\varepsilon>0$, 


$$
\left|4 a_{k} u_{r r}^{-}\left(\delta_{k} \zeta\right) \delta_{k}\left(\zeta u_{r r}^{-}\right)\right| \leq \varepsilon a_{k}\left[\delta_{k}\left(\zeta u_{r r}^{-}\right)\right]^{2}+N \varepsilon^{-1}\left(u_{r r}^{-}\right)^{2}\left(\left|\eta^{\prime}\right|^{2} \zeta+h^{2}\left|\eta^{\prime}\right|^{4}\right),
$$

where

$$
\left(u_{r r}^{-}\right)^{2} h^{2} \leq\left|h u_{r r}\right|^{2}=\left|\left(T_{\ell_{r}}-1\right) u_{-r}\right|^{2},
$$

so that $\left|\eta^{\prime}\right|^{4}\left(u_{r r}^{-}\right)^{2} h^{2} \leq 4\left|\eta^{\prime}\right|^{4} \bar{W}_{r}$ in $Q^{o}$. Therefore, in $Q^{o}$

$$
\left|4 a_{k} u_{r r}^{-}\left(\delta_{k} \zeta\right) \delta_{k}\left(\zeta u_{r r}^{-}\right)\right| \leq \varepsilon a_{k}\left[\delta_{k}\left(\zeta u_{r r}^{-}\right)\right]^{2}+N \varepsilon^{-1}\left|\eta^{\prime}\right|^{2} \zeta\left(u_{r r}^{-}\right)^{2}+N \varepsilon^{-1} \bar{W}_{r}\left|\eta^{\prime}\right|^{4}
$$

Similarly

$$
\left|2 a_{k}\left(u_{r r}^{-}\right)^{2}\left[\zeta \Delta_{k} \zeta-2\left(\delta_{k} \zeta\right)^{2}\right]\right| \leq N\left(\left|\eta^{\prime \prime}\right|+\left|\eta^{\prime}\right|^{2}\right) \zeta\left(u_{r r}^{-}\right)^{2}+N \bar{W}_{r}\left|\eta^{\prime}\right|^{4} .
$$

By Lemma 4.3 of [11] for any $\varepsilon \in(0,1]$

$$
h\left(\delta_{k} \zeta\right)^{2}\left|u_{r r}^{-} \delta_{i} u_{r r}^{-}\right| \leq \varepsilon\left|\delta_{i}\left(\zeta u_{r r}^{-}\right)\right|^{2}+\varepsilon\left|\eta^{\prime}\right|^{2} \zeta\left(u_{r r}^{-}\right)^{2}+N \varepsilon^{-1}\left|\eta^{\prime}\right|^{4}\left[\left(h u_{r r}^{-}\right)^{2}+\left(h^{2} \delta_{i} u_{r r}^{-}\right)^{2}\right] .
$$

Estimate (5.6) leads to

$$
h\left(\delta_{k} \zeta\right)^{2}\left|u_{r r}^{-} \delta_{i} u_{r r}^{-}\right| \leq \varepsilon\left|\delta_{i}\left(\zeta u_{r r}^{-}\right)\right|^{2}+\left|\eta^{\prime}\right|^{2} \zeta\left(u_{r r}^{-}\right)^{2}+N \varepsilon^{-1} \bar{W}_{r}\left|\eta^{\prime}\right|^{4}+N \varepsilon^{-1}\left|\eta^{\prime}\right|^{4}\left(h^{2} \delta_{i} u_{r r}^{-}\right)^{2}
$$

on $Q^{\circ}$, where the last term is estimated by using the fact that $\left|\delta_{i} \psi^{-}\right| \leq\left|\delta_{i} \psi\right|$ for any function $\psi$ implying that

$$
\left|\eta^{\prime}\right|^{4}\left(h^{2} \delta_{i} u_{r r}^{-}\right)^{2} \leq\left|\eta^{\prime}\right|^{4}\left|h \delta_{i}\left(u_{r}+u_{-r}\right)\right|^{2}=\left|\eta^{\prime}\right|^{4}\left|\left(T_{\ell_{i}}-1\right)\left(u_{r}+u_{-r}\right)\right|^{2} \leq N\left|\eta^{\prime}\right|^{4} \bar{W}_{r} .
$$

Hence on $Q^{o}$

$$
h\left(\delta_{k} \zeta\right)^{2}\left|u_{r r}^{-} \delta_{i} u_{r r}^{-}\right| \leq \varepsilon\left|\delta_{i}\left(\zeta u_{r r}^{-}\right)\right|^{2}+\left|\eta^{\prime}\right|^{2} \zeta\left(u_{r r}^{-}\right)^{2}+N \varepsilon^{-1} \bar{W}_{r}\left|\eta^{\prime}\right|^{4} .
$$

Upon combining these estimates, choosing $\varepsilon$ appropriately, and coming back to (5.5), we find on $Q^{\circ}$ that

$$
\begin{aligned}
-2 \zeta^{2} u_{r r}^{-} a_{k} \Delta_{k} u_{r r}^{-} \geq & -a_{k} \Delta_{k}\left(\zeta^{2}\left(u_{r r}^{-}\right)^{2}\right)+a_{k}\left[\delta_{k}\left(\zeta u_{r r}^{-}\right)\right]^{2} \\
& -N\left(\left|\eta^{\prime \prime}\right|+\left|\eta^{\prime}\right|^{2}\right) \zeta\left(u_{r r}^{-}\right)^{2}-N \bar{W}_{r}\left|\eta^{\prime}\right|^{4}
\end{aligned}
$$

Next,

$$
\begin{aligned}
\left.b_{k} \delta_{k}\left(\zeta^{2}\left(u_{r r}^{-}\right)^{2}\right)\right)= & 2 \zeta u_{r r}^{-} b_{k} \delta_{k}\left(\zeta u_{r r}^{-}\right)+b_{k} h\left[\delta_{k}\left(\zeta u_{r r}^{-}\right)\right]^{2} \\
= & 2 \zeta^{2} u_{r r}^{-} b_{k} \delta_{k} u_{r r}^{-}+2 \zeta\left(u_{r r}^{-}\right)^{2} b_{k} \delta_{k} \zeta \\
& +2 h b_{k} u_{r r}^{-} \zeta\left(\delta_{k} \zeta\right) \delta_{k} u_{r r}^{-}+b_{k} h\left[\delta_{k}\left(\zeta u_{r r}^{-}\right)\right]^{2} .
\end{aligned}
$$

Here $\left|\delta_{k} \zeta\right| \leq 2\left|\eta^{\prime}\right|$, since $|\eta| \leq 1$. Also $a_{k} \geq \delta$, so that

$$
\left|2 \zeta\left(u_{r r}^{-}\right)^{2} b_{k} \delta_{k} \zeta\right| \leq N^{*}\left|\eta^{\prime}\right| \zeta a_{k} u_{k r}^{2}
$$


Furthermore, $h T_{\ell_{k}} u_{r r}^{-}=T_{\ell_{k}}\left(u_{r}+u_{-r}\right)^{-}$, implying that on $Q^{o}$

$$
\begin{aligned}
\left|2 h b_{k} u_{r r}^{-} \zeta\left(\delta_{k} \zeta\right) \delta_{k} u_{r r}^{-}\right| & =\left|2 h b_{k} u_{r r}^{-}\left(\delta_{k} \zeta\right)\left[\delta_{k}\left(\zeta u_{r r}^{-}\right)-\left(\delta_{k} \zeta\right) T_{\ell_{k}} u_{r r}^{-}\right]\right| \\
& \leq N^{*}\left|\eta^{\prime}\right| \bar{W}_{r}^{1 / 2} a_{k}^{1 / 2}\left|\delta_{k}\left(\zeta u_{r r}^{-}\right)\right|+N^{*} u_{r r}^{-} \sum_{k}\left(\delta_{k} \zeta\right)^{2} \bar{W}_{r}^{1 / 2},
\end{aligned}
$$

where, owing to the inequality $h\left|\eta^{\prime}\right| \leq 2$, the last term is dominated by

$$
\begin{aligned}
N^{*} \bar{W}_{r}^{1 / 2} u_{r r}^{-}\left(\zeta\left|\eta^{\prime}\right|^{2}+h^{2}\left|\eta^{\prime}\right|^{4}\right) \leq & \left(N^{*} \zeta^{1 / 2}\left|\eta^{\prime}\right|^{3 / 2} \bar{W}_{r}^{1 / 2}\right)\left(\left|\eta^{\prime}\right|^{1 / 2} \zeta^{1 / 2} u_{r r}^{-}\right) \\
& +N^{*}\left|\eta^{\prime}\right|^{3} \bar{W}_{r} \leq N^{*}\left|\eta^{\prime}\right| \zeta a_{k} u_{k r}^{2}+N^{*}\left|\eta^{\prime}\right|^{3} \bar{W}_{r} .
\end{aligned}
$$

Hence, in $Q^{o}$

$$
\begin{aligned}
-2 \zeta^{2} u_{r r}^{-} b_{k} \delta_{k} u_{r r}^{-} \geq & \left.-b_{k} \delta_{k}\left(\zeta^{2}\left(u_{r r}^{-}\right)^{2}\right)\right)-N^{*}\left|\eta^{\prime}\right| \zeta a_{k} u_{k r}^{2} \\
& -\left(N^{*} h+1 / 2\right) a_{k}\left[\delta_{k}\left(\zeta u_{r r}^{-}\right)\right]^{2}-N^{*}\left(\left|\eta^{\prime}\right|^{2}+\left|\eta^{\prime}\right|^{3}\right) \bar{W}_{r} .
\end{aligned}
$$

For $N^{*} h \leq 1 / 2$ estimates (5.7) and (5.8) and the fact that $\left|\eta^{\prime}\right|^{3} \leq\left|\eta^{\prime}\right|^{2}+\left|\eta^{\prime}\right|^{4}$ lead to (5.3).

To prove (5.4) observe that (recall that $\delta_{k} u_{r}=-u_{-k r}$ and $a_{-k}=a_{k}$ )

$$
a_{k} \Delta_{k}\left(\zeta u_{r}^{2}\right)=a_{k} \zeta\left[2 u_{r} \Delta_{k} u_{r}+2 u_{k r}^{2}\right]+a_{k} u_{r}^{2} \Delta_{k} \zeta+2 a_{k}\left(\delta_{k} \zeta\right)\left(h u_{-k r}^{2}-2 u_{r} u_{-k r}\right),
$$

where

$$
u_{r}^{2}\left|\Delta_{k} \zeta\right| \leq N\left(\left|\eta^{\prime \prime}\right|+\left|\eta^{\prime}\right|^{2}\right) \bar{W}_{r}
$$

and owing to (5.6)

$$
\begin{aligned}
\left|2 a_{k}\left(\delta_{k} \zeta\right)\left(h u_{-k r}^{2}-2 u_{r} u_{-k r}\right)\right| & \leq N a_{k}\left(\left|\eta^{\prime}\right| \zeta^{1 / 2}+h\left|\eta^{\prime}\right|^{2}\right)\left(\left|u_{r} u_{k r}\right|+h u_{k r}^{2}\right) \\
& \leq N a_{k}\left|u_{k r}\right|\left(\left|\eta^{\prime}\right| \zeta^{1 / 2}+h\left|\eta^{\prime}\right|^{2}\right) \bar{W}_{r}^{1 / 2} \\
& \leq N a_{k}\left|u_{k r}\right|\left|\eta^{\prime}\right| \zeta^{1 / 2} \bar{W}_{r}^{1 / 2}+N\left|\eta^{\prime}\right|^{2} \bar{W}_{r} \\
& \leq(1 / 2) \zeta a_{k} u_{k r}^{2}+N\left|\eta^{\prime}\right|^{2} \bar{W}_{r} .
\end{aligned}
$$

It follows that in $Q^{\circ}$

$$
-2 \zeta a_{k} u_{r} \Delta_{k} u_{r} \geq-a_{k} \Delta_{k}\left(\zeta u_{r}^{2}\right)+(3 / 2) \zeta a_{k} u_{k r}^{2}-N\left(\left|\eta^{\prime \prime}\right|+\left|\eta^{\prime}\right|^{2}\right) \bar{W}_{r} .
$$

Also

$$
b_{k} \delta_{k}\left(\zeta u_{r}^{2}\right)=2 \zeta u_{r} b_{k} \delta_{k} u_{r}+\zeta b_{k} h u_{-k r}^{2}+u_{r}^{2} b_{k} \delta_{k} \zeta+h b_{k}\left(\delta_{k} \zeta\right)\left[h u_{-k r}^{2}-2 u_{r} u_{-k r}\right] .
$$

Here in $Q^{o}$

$$
\left|u_{r}^{2} b_{k} \delta_{k} \zeta\right| \leq N^{*}\left|\eta^{\prime}\right| W_{r}, \quad\left|h b_{k}\left(\delta_{k} \zeta\right)\left[h u_{-k r}^{2}-2 u_{r} u_{-k r}\right] \leq N^{*}\right| \eta^{\prime} \mid \bar{W}_{r},
$$

where the last estimate follows from an equality similar to (5.6). Furthermore, 


$$
\left|\zeta b_{k} h u_{-k r}^{2}\right| \leq(1 / 2) \zeta a_{k} u_{k r}^{2}
$$

if $N^{*} h \leq 1$ and $N^{*}$ is chosen appropriately.

Upon combining this estimates with (5.9) we come to (5.4) and the lemma is proved.

For a constant $\nu \geq 0$ introduce an operator (recall that $r$ is fixed)

$$
L_{\nu} \phi=\zeta^{2} u_{r r}^{-} \Delta_{r} \phi-\nu \zeta u_{r} \delta_{r} \phi
$$

Observe that

$$
L_{\nu} u=-\zeta^{2}\left(u_{r r}^{-}\right)^{2}-\nu \zeta u_{r}^{2}=:-V_{\nu} .
$$

Lemma 5.3. There exists $N=N(m, \delta) \geq 1$ and $N^{*}=N^{*}(m, \delta)$ such that if

$$
\nu \geq N^{*}\left\|\eta^{\prime}\right\|+N\left(\left\|\eta^{\prime \prime}\right\|+\left\|\eta^{\prime}\right\|^{2}\right)
$$

and $N^{*} h \leq 1$, then on $Q^{o}$ for any $\alpha=(a, b, c) \in A$ we have

$$
2 L_{\nu}\left[a_{k} \Delta_{k}+b_{k} \delta_{k}\right] u \geq-\left[a_{k} \Delta_{k}+b_{k} \delta_{k}\right] V_{\nu}-\left(N \nu^{2}+N^{*} \nu\right) \bar{W}_{r}+(\nu / 2) \zeta a_{k} u_{k r}^{2} .
$$

Furthermore, $N^{*}=0$ if $b \equiv 0$.

Proof. Since, for each $k$, the operators $a_{k} \Delta_{k}+b_{k} \delta_{k}$ respect the maximum principle, it follows by Lemma 4.2 of [11] that

$$
u_{r r}^{-}\left(a_{k} \Delta_{k}+b_{k} \delta_{k}\right) u_{r r} \geq-u_{r r}^{-}\left[a_{k} \Delta_{k}+b_{k} \delta_{k}\right] u_{r r}^{-} .
$$

Hence,

$$
I:=\zeta^{2} u_{r r}^{-} \Delta_{r}\left[a_{k} \Delta_{k}+b_{k} \delta_{k}\right] u=\zeta^{2} u_{r r}^{-}\left[a_{k} \Delta_{k}+b_{k} \delta_{k}\right] u_{r r} \geq-\zeta^{2} u_{r r}^{-}\left[a_{k} \Delta_{k}+b_{k} \delta_{k}\right] u_{r r}^{-},
$$

which by Lemma 5.2 and the observation that

$$
\zeta\left(u_{r r}^{-}\right)^{2} \leq N \zeta a_{k} u_{k r}^{2},
$$

for $N^{*} h \leq 1$ yields

$$
\begin{aligned}
2 I \geq & -\left[a_{k} \Delta_{k}+b_{k} \delta_{k}\right]\left(\zeta^{2}\left(u_{r r}^{-}\right)^{2}\right)-N\left(N^{*}\left|\eta^{\prime}\right|+\left|\eta^{\prime \prime}\right|+\left|\eta^{\prime}\right|^{2}\right) \zeta a_{k} u_{k r}^{2} \\
& -\left(N^{*}\left|\eta^{\prime}\right|^{2}+N\left|\eta^{\prime}\right|^{4}\right) \bar{W}_{r} .
\end{aligned}
$$

Furthermore, by Lemma 5.2

$$
\begin{aligned}
-2 \nu \zeta u_{r} \delta_{r}\left[a_{k} \Delta_{k}+b_{k} \delta_{k}\right] u= & -2 \nu \zeta u_{r}\left[a_{k} \Delta_{k}+b_{k} \delta_{k}\right] u_{r} \\
\geq & -\left[a_{k} \Delta_{k}+b_{k} \delta_{k}\right]\left(\nu \zeta u_{r}^{2}\right)+\nu \zeta a_{k} u_{k r}^{2} \\
& -N \nu\left(\left|\eta^{\prime \prime}\right|+\left|\eta^{\prime}\right|^{2}+N^{*}\left|\eta^{\prime}\right|\right) \bar{W}_{r} .
\end{aligned}
$$


By combining this with (5.13) and recalling (5.10) we find

$$
\begin{aligned}
2 L_{\nu}\left[a_{k} \Delta_{k}+b_{k} \delta_{k}\right] u \geq & -\left[a_{k} \Delta_{k}+b_{k} \delta_{k}\right] V_{\nu} \\
& +\left(\nu-N_{1}^{*}\left|\eta^{\prime}\right|-N_{1}\left|\eta^{\prime \prime}\right|-N_{1}\left|\eta^{\prime}\right|^{2}\right) \zeta a_{k} u_{k r}^{2} \\
& -\left[N^{*}\left(\left|\eta^{\prime}\right|^{2}+\nu\left|\eta^{\prime}\right|\right)+N\left(\left|\eta^{\prime}\right|^{4}+\nu\left|\eta^{\prime \prime}\right|+\nu\left|\eta^{\prime}\right|^{2}\right)\right] \bar{W}_{r} .
\end{aligned}
$$

We may assume that $N_{1} \geq 1$ and then, if

$$
\nu \geq 2 N_{1}^{*}\left|\eta^{\prime}\right|+2 N_{1}\left(\left|\eta^{\prime \prime}\right|+\left|\eta^{\prime}\right|^{2}\right)
$$

we have that $\left|\eta^{\prime}\right|^{2} \leq \nu,\left|\eta^{\prime \prime}\right| \leq \nu$, and

$$
\left|\eta^{\prime}\right|^{4}+\nu\left|\eta^{\prime \prime}\right|+\nu\left|\eta^{\prime}\right|^{2} \leq 3 \nu^{2}
$$

Also

$$
N^{*}\left(\left|\eta^{\prime}\right|^{2}+\nu\left|\eta^{\prime}\right|\right) \leq N^{*}\left(\nu+\nu^{3 / 2}\right) \leq N^{*}\left(\nu+\nu^{2}\right) \leq N^{*} \nu+N \nu^{2} .
$$

After that (5.14) clearly yields (5.12) and the lemma is proved.

Proof of Theorem 5.1. Denote by $N_{0}$ and $N_{0}^{*}$ the constants $N$ and $N^{*}$ in Lemma 5.3 and take and fix a $\nu$ satisfying (5.11) (with $N_{0}$ and $N_{0}^{*}$ in place of $N$ and $N^{*}$ ).

Observe that (5.2) obviously holds on $Q \backslash Q^{\circ}$ and we only need to prove it on $Q^{o}$. Also notice that

$$
\left|u_{r r}\right|=h^{-1}\left|u_{r}+u_{-r}\right| \leq 2 h^{-1} \bar{W}_{r}^{1 / 2},
$$

which shows that (5.2) holds if $h \geq \nu^{-1 / 2}$ or if $N_{0}^{*} h \geq 1$. Therefore below we assume that

$$
h \leq \nu^{-1 / 2}, \quad N_{0}^{*} h \leq 1
$$

Introduce

$$
D^{o}=\left\{x \in Q^{o}: \zeta(x) u_{r r}^{-}(x) \geq \nu h u_{r}(x)\right\} .
$$

If $x \in Q^{o} \backslash D^{o}$, then $\zeta(x) u_{r r}^{-}(x) \leq \nu h u_{r}(x)$ and (5.2) holds at $x$ in light of (5.15). Therefore, in the remaining part of the proof we concentrate on establishing (5.2) for $x \in D^{o}$, assuming, of course, that $D^{o} \neq \emptyset$.

Denote

$$
D=D^{o} \cup\left\{x+h \Lambda: x \in D^{o}\right\} \quad(\subset Q) .
$$

If $V_{\nu}$ in $D^{o}$ is less than its maximum over $D \backslash D^{o}$, then in $D^{o}$

$$
\zeta^{2}\left[u_{r r}^{-}\right]^{2} \leq V_{\nu} \leq \max \left[\max _{Q \backslash Q^{\circ}} \zeta^{2}\left[u_{r r}^{-}\right]^{2}, \max _{Q^{o} \backslash D^{o}} \zeta^{2}\left[u_{r r}^{-}\right]^{2}\right]+\nu \bar{W}_{r}
$$

where the maximums are less than the right-hand side of (5.2) by the above. Hence, in the rest of the proof we consider the case that the maximum over $D$ of $V_{\nu}$ is attained at a point $x_{0} \in D^{o}$. 
Notice that if a function $\phi(x)$ is such that $\phi(x) \leq \phi\left(x_{0}\right)$ for $x \in x_{0}+h \Lambda$, then at $x_{0}$

$$
\begin{aligned}
h^{2} L_{\nu} \phi\left(x_{0}\right) & =\zeta\left[\phi\left(x_{0}+h \ell_{r}\right)\left(\zeta u_{r r}^{-}-\nu h u_{r}\right)+\phi\left(x_{0}-h \ell_{r}\right) \zeta u_{r r}^{-}\right]-\zeta\left[2 \zeta u_{r r}^{-}-\nu h u_{r}\right] \phi \\
& \leq \zeta\left[\left(\zeta u_{r r}^{-}-\nu h u_{r}\right) \phi+\zeta u_{r r}^{-} \phi\right]-\zeta\left[2 \zeta u_{r r}^{-}-\nu h u_{r}\right] \phi
\end{aligned}
$$

where the last expression is zero. Thus

$$
L_{\nu} \phi\left(x_{0}\right) \leq 0
$$

which in the terminology from [11] means that $L_{\nu}$ respects the maximum principle.

Next, we can find an $\bar{\alpha}=(\bar{a}, \bar{b}, \bar{c}) \in A$ such that

$$
\bar{a}_{k} \Delta_{k} u\left(x_{0}\right)+\bar{b}_{k} \delta_{k} u\left(x_{0}\right)-\bar{c} u\left(x_{0}\right)+f\left(\bar{\alpha}, x_{0}\right)=\mathcal{P}[u]\left(x_{0}\right)=0 .
$$

Since $\mathcal{P}[u] \leq 0$ in $Q$, we have that

$$
\phi(x):=\bar{a}_{k} \Delta_{k} u(x)+\bar{b}_{k} \delta_{k} u(x)-\bar{c} u(x)+f(\bar{\alpha}, x) \leq 0
$$

for $x \in x_{0}+h \Lambda$. Hence,

$$
0 \geq 2 L_{\nu} \phi\left(x_{0}\right)=\bar{a}_{k} 2 L_{\nu} \Delta_{k} u\left(x_{0}\right)+\bar{b}_{k} 2 L_{\nu} \delta_{k} u\left(x_{0}\right)-\bar{c} 2 L_{\nu} u\left(x_{0}\right)+2 L_{\nu} f(\bar{\alpha}, \cdot)\left(x_{0}\right),
$$

which owing to (5.10) and (5.12) yields

$0 \leq\left[\bar{a}_{k} \Delta_{k}+\bar{b}_{k} \delta_{k}-2 \bar{c}\right] V_{\nu}\left(x_{0}\right)-(\nu / 2) \zeta \bar{a}_{k} u_{k r}^{2}\left(x_{0}\right)+\left(N \nu^{2}+N^{*} \nu\right) \bar{W}_{r}-2 L_{\nu} f(\bar{\alpha}, \cdot)\left(x_{0}\right)$.

Here the last term is dominated by

$$
K_{2} \zeta^{2} u_{r r}^{-}\left(x_{0}\right)+\nu\left|u_{r}\left(x_{0}\right)\right| K_{1} \leq N \nu^{-1} K_{2}^{2}+(\nu / 4) \zeta \bar{a}_{k} u_{k r}^{2}\left(x_{0}\right)+K_{1}^{2}+\nu^{2} \bar{W}_{r} .
$$

Furthermore, by the maximum principle

$$
\left[\bar{a}_{k} \Delta_{k}+\bar{b}_{k} \delta_{k}-2 \bar{c}\right] V_{\nu}\left(x_{0}\right) \leq 0,
$$

since $V_{\nu} \geq 0$ attains its maximum at $x_{0}$.

We now conclude that

$$
(\nu / 4) \zeta \bar{a}_{k} u_{k r}^{2}\left(x_{0}\right) \leq\left(N \nu^{2}+N^{*} \nu\right) \bar{W}_{r}+N \nu^{-1} K_{2}^{2}+K_{1}^{2},
$$

which implies that in $D^{o}$

$$
\zeta^{2}\left(u_{r r}^{-}\right)^{2} \leq V_{\nu}\left(x_{0}\right) \leq N \zeta \bar{a}_{k} u_{k r}^{2}\left(x_{0}\right)+\nu \bar{W}_{r} \leq\left(N \nu+N^{*}\right) \bar{W}_{r}+N \nu^{-2} K_{2}^{2}+\nu^{-1} K_{1}^{2} .
$$

Thus, estimate (5.2) holds on $D^{o}$ as well and this proves the theorem.

6. A model cut-off equation. We will work in the setting of Section 5. However now $h>0$ is not fixed. Take a function $\mathcal{H}(u, x)$, where $x \in \mathbb{R}^{d}, u=\left(u^{\prime}, u^{\prime \prime}\right) \in$ $\mathbb{R}^{1+m^{\prime}+2 m}$.

Assumption 6.1. (i) The function $\mathcal{H}$ is Lipschitz continuous in $u$ for every $x$, and at all points of differentiability of $\mathcal{H}$ with respect to $u$ we have

$$
\delta \leq \mathcal{H}_{u_{k}^{\prime \prime}} \leq \delta^{-1}, \quad k= \pm 1, \ldots, \pm m, \quad \delta \leq-\mathcal{H}_{u_{0}^{\prime}} \leq \delta^{-1}
$$




$$
\left|\mathcal{H}_{u_{k}^{\prime}}\right| \leq \delta^{-1}, \quad k=1, \ldots, m^{\prime}
$$

(ii) The number $\overline{\mathcal{H}}=\sup _{x}|\mathcal{H}(0,0, x)|$ is finite;

(iii) The function $\mathcal{H}$ is locally Lipschitz continuous in $x$ for every $u$ and there exists a constant $N^{\prime}$ such that at all points of differentiability of $\mathcal{H}$ with respect to $x$ we have

$$
\left|\mathcal{H}_{x_{i}}(u, x)\right| \leq N^{\prime}(1+|u|), \quad \forall i ;
$$

(iv) We have $\operatorname{Span}\left(\ell_{1}, \ldots, \ell_{m}\right)=\mathbb{R}^{d}$.

Define

$$
\begin{aligned}
\mathcal{P}\left(u^{\prime}, u^{\prime \prime}, x\right)= & \mathcal{P}\left(u^{\prime}, u^{\prime \prime}\right) \\
= & 2 \delta^{-1} \sum_{k}\left(u_{k}^{\prime \prime}\right)^{+}-(\delta / 2) \sum_{k}\left(u_{k}^{\prime \prime}\right)^{-} \\
& +2 \delta^{-1} \sum_{k \geq 1}\left|u_{k}^{\prime}\right|-(\delta / 2)\left(u_{0}^{\prime}\right)^{+}+2 \delta^{-1}\left(u_{0}^{\prime}\right)^{-} \\
= & \max _{\substack{\delta / 2 \leq a_{k} \leq 2 / \delta \\
|k|=1, \ldots, m}} \max _{|k| b_{k} \mid \leq 2 / \delta} \max _{|k|, \ldots, m^{\prime}}\left[\begin{array}{c}
\delta \leq c \leq 2 / \delta \\
|i|=1
\end{array}\right.
\end{aligned}
$$

For functions $v(x)$ introduce

$$
H[v](x)=\mathcal{H}\left(v(x), \partial v(x), \partial^{2} v(x), x\right)
$$

whenever and wherever it makes sense, where

$$
\begin{gathered}
\partial v=\left(v_{\left(\ell_{1}\right)}, \ldots, v_{\left(\ell_{m^{\prime}}\right)}\right), \\
\partial^{2} v=\left(v_{\left(\ell_{-m}\right)\left(\ell_{-m}\right)}, \ldots, v_{\left(\ell_{-1}\right)\left(\ell_{-1}\right)}, v_{\left(\ell_{1}\right)\left(\ell_{1}\right)}, \ldots, v_{\left(\ell_{m}\right)\left(\ell_{m}\right)}\right),
\end{gathered}
$$

and $v_{(\ell)}=\ell_{i} v_{x_{i}}, v_{(\ell)(\ell)}=\ell_{i} \ell_{j} v_{x_{i} x_{j}}$. Similarly,

$$
P[v](x)=\mathcal{P}\left(v(x), \partial v(x), \partial^{2} v(x)\right) .
$$

Let $\Omega$ be a bounded $C^{2}$ domain in $\mathbb{R}^{d}, g$ be a bounded function on $\mathbb{R}^{d}$ such that $g \in C^{1,1}(\bar{\Omega})$, and let $K \geq 0$ be a finite number.

TheOREM 6.1. In addition to Assumption 6.1 suppose that $\pm e_{i}, \pm\left(e_{i}+e_{j}\right), e_{i}-$ $e_{j} \in \Lambda, i, j=1, . ., d$, were $e_{1}, \ldots, e_{d}$ is the standard orthonormal basis in $\mathbb{R}^{d}$ and assume that all vectors in $\Lambda$ have rational coordinates. Then there exists a unique $v \in C^{0,1}(\bar{\Omega}) \cap C_{\text {loc }}^{1,1}(\Omega)$ such that $v=g$ on $\partial \Omega$ and

$$
H_{K}[v]=0
$$

(a.e.) in $\Omega$, where

$$
H_{K}[v]=\max (H[v], P[v]-K)
$$

Furthermore,

$$
|v|,\left|D_{i} v\right|, \rho\left|D_{i j} v\right| \leq N\left(\overline{\mathcal{H}}+K+\|g\|_{C^{1,1}(\Omega)}\right)
$$


in $\Omega$ (a.e.) for all $i, j$, where $N$ is a constant depending only on $\Omega,\left\{\ell_{1}, \ldots, \ell_{m}\right\}, d$, and $\delta$ (but not on $N^{\prime}$ ).

To prove the theorem, we are going to use finite-difference approximations of the operators $H[v]$ and $P[v]$. For $h>0$ introduce

$$
P_{h}[v](x)=\mathcal{P}\left(v(x), \delta_{h} v(x), \delta_{h}^{2} v(x)\right),
$$

where naturally

$$
\begin{gathered}
\delta_{h} u=\left(\delta_{h, 1} u, \ldots, \delta_{h, m^{\prime}} u\right), \\
\delta_{h}^{2} u=\left(\Delta_{h,-m} u, \ldots, \Delta_{h,-1} u, \Delta_{h, 1} u, \ldots, \Delta_{h, m} u\right) .
\end{gathered}
$$

Similarly we introduce $H_{h}$ and $H_{K, h}$.

Lemma 6.2. Under Assumptions 6.1 (i), (ii)

$$
\mathcal{H} \leq \mathcal{P}-(\delta / 2)\left(\sum_{k}\left|u_{k}^{\prime \prime}\right|+\sum_{k}\left|u_{k}^{\prime}\right|\right)+\overline{\mathcal{H}}
$$

Proof. Basically, we just repeat part of the proof of Lemma 3.1. From Hadamard's formula

$$
\begin{aligned}
\mathcal{H}\left(u^{\prime}, u^{\prime \prime}, x\right)-H(0,0, x)= & u_{k}^{\prime \prime} \int_{0}^{1} \mathcal{H}_{u_{k}^{\prime \prime}}\left(t u^{\prime}, t u^{\prime \prime}, x\right) d t \\
& +\sum_{k \geq 1} u_{k}^{\prime} \int_{0}^{1} \mathcal{H}_{u_{k}^{\prime}}\left(t u^{\prime}, t u^{\prime \prime}, x\right) d t+u_{0}^{\prime} \int_{0}^{1} \mathcal{H}_{u_{0}^{\prime}}\left(t u^{\prime}, t u^{\prime \prime}, x\right) d t
\end{aligned}
$$

we obtain

$$
\begin{aligned}
\mathcal{H}\left(u^{\prime}, u^{\prime \prime}, x\right)-\mathcal{H}(0,0, x) \leq & \delta^{-1} \sum_{k}\left(u_{k}^{\prime \prime}\right)^{+}-\delta \sum_{k}\left(u_{k}^{\prime \prime}\right)^{-}+\delta^{-1} \sum_{k \geq 1}\left|u_{k}^{\prime}\right| \\
& -\delta\left(u_{0}^{\prime}\right)^{+}+\delta^{-1}\left(u_{0}^{\prime}\right)^{-} \\
= & \mathcal{P}\left(u^{\prime}, u^{\prime \prime}\right)-\delta^{-1} \sum_{k}\left(u_{k}^{\prime \prime}\right)^{+}-(\delta / 2) \sum_{k}\left(u_{k}^{\prime \prime}\right)^{-}-\delta^{-1} \sum_{k \geq 1}\left|u_{k}^{\prime}\right| \\
& -(\delta / 2)\left(u_{0}^{\prime}\right)^{+}-\delta^{-1}\left(u_{0}^{\prime}\right)^{-}
\end{aligned}
$$

and (6.4) follows. The lemma is proved.

Introduce $B$ as the smallest closed ball containing $\Lambda$ and set

$$
\Omega_{h}=\{x \in \Omega: x+h B \subset \Omega\}=\{x: \rho(x)>\lambda h\},
$$

where $\lambda$ is the radius of $B$.

Consider the equation

$$
H_{K, h}[v]=0 \quad \text { in } \quad \Omega_{h}
$$

with boundary condition

$$
v=g \quad \text { on } \quad \mathbb{R}^{d} \backslash \Omega_{h} .
$$


It is a rather simple fact that for sufficiently small $h>0$ there exists a unique bounded solution $v=v_{h}$ of (6.5)-(6.6) (see, for instance, [13] or Theorem 8.2 in [10] or else Theorem 2.2 in [12]). By the way, we do not include $K$ in the notation $v_{h}$ since $K$ is a fixed number.

Below by $h_{0}$ and $N$ with occasional indices we denote various (finite) constants depending only on $\Omega,\left\{\ell_{1}, \ldots, \ell_{m}\right\}, d$, and $\delta$.

In the following lemma the additional assumption of Theorem 6.1 concerning the $e_{i}$ 's and the $\ell_{k}$ 's is not used.

Lemma 6.3. Under Assumptions 6.1 (i), (ii), (iv) there are constants $h_{0}>0$ and $N$ such that for all $h \in\left(0, h_{0}\right]$ and $|r|=1, \ldots, m$

$$
\begin{aligned}
\left|v_{h}-g\right| & \leq N\left(\overline{\mathcal{H}}+K+\|g\|_{C^{1,1}(\Omega)}\right) \rho, \\
\left|\delta_{h, r} v_{h}\right| & \leq N\left(\overline{\mathcal{H}}+K+\|g\|_{C^{1,1}(\Omega)}\right)
\end{aligned}
$$

on $\Omega_{h}$.

Proof. Introduce

$$
\mathcal{H}_{K}=\max (\mathcal{H}, \mathcal{P}-K) .
$$

Clearly, $\mathcal{H}_{K}$ satisfies Assumption 6.1 with $\delta / 2$ in place of $\delta$. Therefore, by Hadamard's formula there exist functions $a_{k}, b_{k}, k= \pm 1, \ldots, \pm m$, and $c$ such that

$$
\delta / 2 \leq a_{k} \leq 2 \delta^{-1}, \quad\left|b_{k}\right| \leq 2 \delta^{-1}, \quad \delta / 2 \leq c \leq 2 \delta^{-1}
$$

and in $\Omega_{h}$ we have

$$
\begin{aligned}
-H_{K}[0] & =H_{K, h}\left[v_{h}\right]-H_{K}[0] \\
& =a_{k} \Delta_{h, k} v_{h}+b_{k} \delta_{h, k} v_{h}-c v_{h} \\
& =a_{k} \Delta_{h, k}\left(v_{h}-g\right)+b_{k} \delta_{h, k}\left(v_{h}-g\right)-c\left(v_{h}-g\right)+f,
\end{aligned}
$$

where

$$
f=a_{k} \Delta_{h, k} g+b_{k} \delta_{h, k} g-c g .
$$

After that (6.7) is proved by using the barrier function $\Phi$ from Lemma 2.4 of [12] (cf. Lemma 2.5 in [12]). It implies that

$$
\left|v_{h}-g\right| \leq N_{1}\left(\overline{\mathcal{H}}+K+\|g\|_{C^{1,1}(\Omega)}\right) h \quad \text { on } \quad \Omega \backslash \Omega_{3 h} .
$$

To prove (6.8), fix an $r$ and define

$$
Q^{o}=\left\{x \in \Omega_{2 h}:(\delta / 2)\left|\delta_{h, r} v_{h}\right| \geq \overline{\mathcal{H}}+K\right\} .
$$

If $Q^{o}=\emptyset$, then $(\delta / 2)\left|\delta_{h, r} v_{h}\right| \leq \overline{\mathcal{H}}+K$ in $\Omega_{2 h}$, and by virtue of (6.10),

$$
\left|\delta_{h, r}\left(v_{h}-g\right)\right| \leq 2 N_{1}\left(\overline{\mathcal{H}}+K+\|g\|_{C^{1,1}(\Omega)}\right)
$$

in $\Omega_{h} \backslash \Omega_{2 h}$. In that case (6.8) obviously holds.

Therefore, we assume that $Q^{o} \neq \emptyset$ and owing to Lemma 6.2 conclude that

$$
P_{h}\left[v_{h}\right]=K
$$


in $Q^{\circ}$. Furthermore, (6.5) implies that

$$
P_{h}\left[v_{h}\right] \leq K
$$

in $\Omega_{h}$.

Now use again the mean value theorem to conclude that

$$
\delta_{h, r} P_{h}\left[v_{h}\right]=a_{k} \Delta_{h, k}\left(\delta_{h, r} v_{h}\right)+b_{k} \delta_{h, k}\left(\delta_{h, r} v_{h}\right)-c\left(\delta_{h, r} v_{h}\right)
$$

for some functions $a_{k}(x), b_{k}(x), c(x)$ satisfying (6.9). In addition, $\delta_{h, r} P_{h}\left[v_{h}\right] \leq 0$ in $Q^{o}$ owing to $(6.11)$ and $(6.12)$, that is in $Q^{o}$

$$
a_{k} \Delta_{h, k}\left(\delta_{h, r} v_{h}\right)+b_{k} \delta_{h, k}\left(\delta_{h, r} v_{h}\right)-c\left(\delta_{h, r} v_{h}\right) \leq 0 .
$$

For small enough $h_{0}$ the operator $a_{k} \Delta_{h, k}+b_{k} \delta_{h, k}-c$ with $h \in\left(0, h_{0}\right]$ respects the maximum principle and therefore in $Q^{\circ}$ (see Theorem 2.2 in [12])

$$
\left(\delta_{h, r} v_{h}\right)^{-} \leq \sup _{\Omega_{h} \backslash Q^{o}}\left(\delta_{h, r} v_{h}\right)^{-} .
$$

While estimating the right-hand side of (6.13), notice that if $x \in \Omega_{h} \backslash Q^{o}$, then either $x \notin \Omega_{2 h}$, in which case (6.8) holds by the above, or else $x \in \Omega_{2 h}$ but $(\delta / 2)\left|\delta_{h, r} v_{h}\right| \leq$ $\overline{\mathcal{H}}+K$. It follows that the right-hand side of (6.13) is dominated by the right-hand side of $(6.8)$, if $h \in\left(0, h_{0}\right]$ and $h_{0}>0$ is sufficiently small.

Thus, in all cases

$$
\left(\delta_{h, r} v_{h}\right)^{-} \leq N\left(\overline{\mathcal{H}}+K+\|g\|_{C^{1,1}(\Omega)}\right)
$$

on $\Omega_{h}$. Upon replacing here $r$ with $-r$, we get

$$
T_{h,-\ell_{r}}\left(\delta_{h, r} v_{h}\right)^{+} \leq N\left(\overline{\mathcal{H}}+K+\|g\|_{C^{1,1}(\Omega)}\right)
$$

in $\Omega_{h}$, which after being combined with the previous estimate proves (6.8) in $\Omega_{2 h}$. In $\Omega_{h} \backslash \Omega_{2 h}$ estimate (6.8) has been established above. The lemma is proved.

LEMma 6.4. Suppose that Assumptions 6.1 (i), (ii), (iv) are satisfied. Assume also that all vectors in $\Lambda$ have rational coordinates. Then there are constants $h_{0}>0$ and $N$ such that for all $h \in\left(0, h_{0}\right]$ and $|r|=1, \ldots, m$

$$
(\rho-6 \lambda h)\left|\Delta_{h, r} v_{h}\right| \leq N\left(\overline{\mathcal{H}}+K+\|g\|_{C^{1,1}(\Omega)}\right)
$$

on $\mathbb{R}^{d}$ (we remind the reader that $\lambda$ is the radius of $B$ ).

Proof. Clearly, the assertion of the lemma would follow if we can prove that (6.14) holds on $y+h \Lambda_{\infty}$ for any $y \in \mathbb{R}^{d}$ with a constant $N$ independent of $h$ and $y$. Without losing generality we concentrate on $y=0$. Then for a fixed $r$ define

$$
Q^{o}:=\left\{x \in\left(h \Lambda_{\infty}\right) \cap \Omega_{3 h}:(\delta / 2)\left|\Delta_{h, r} v_{h}(x)\right| \geq \overline{\mathcal{H}}+K\right\} .
$$

If $x \in h \Lambda_{\infty}$ is such that $x \notin Q^{o}$, then either $x \notin \Omega_{3 h}$, so that $\rho(x) \leq 3 \lambda h$ and (6.14) holds, or else $x \in \Omega_{3 h}$ but $(\delta / 2)\left|\Delta_{h, r} v_{h}(x)\right| \leq \overline{\mathcal{H}}+K$, in which case (6.14) holds again. define

Thus we need only prove (6.14) on $Q^{o}$ assuming, of course, that $Q^{o} \neq \emptyset$. Then

$$
Q=\left\{x+h \Lambda: x \in Q^{o}\right\}
$$


Observe that $Q$ is a finite set since $\ell_{k}$ have rational coordinates and there is a number $M$ such that the coordinates of all points in $M \Lambda_{1, \infty}$ are integers and the number of points with integral coordinates lying in a bounded domain is finite.

Next by Lemma 6.2 we have that (6.11) holds in $Q^{o}$ and (6.12) holds in $Q \backslash Q^{o}$.

To proceed further observe a standard fact that there are constants $\mu_{0}>0$ and $N \in[0, \infty)$ depending only on $\Omega$ such that for any $\mu \in\left(0, \mu_{0}\right]$ there exists an $\eta_{\mu} \in$ $C_{0}^{\infty}(\Omega)$ satisfying

$$
\begin{gathered}
\eta_{\mu}=1 \quad \text { on } \quad \Omega_{2 \mu}, \quad \eta_{\mu}=0 \quad \text { outside } \quad \Omega_{\mu}, \\
\left|\eta_{\mu}\right| \leq 1, \quad\left|D \eta_{\mu}\right| \leq N / \mu, \quad\left|D^{2} \eta_{\mu}\right| \leq N / \mu^{2} .
\end{gathered}
$$

By Theorem 5.1 and Lemma 6.3 there are constants $N$ and $h_{0}>0$ such that, for any number $\nu$ satisfying

$$
\nu \geq N\left(\left\|\eta_{\mu}^{\prime}\right\|_{h}+\left\|\eta_{\mu}^{\prime}\right\|_{h}^{2}+\left\|\eta_{\mu}^{\prime \prime}\right\|_{h}\right)
$$

we have in $Q^{o}$ that

$$
\eta_{\mu}^{4}\left[\left(\Delta_{r} v_{h}\right)^{-}\right]^{2} \leq \max _{Q \backslash Q^{o}} \eta_{\mu}^{4}\left[\left(\Delta_{r} v_{h}\right)^{-}\right]^{2}+N(\nu+1)\left(\overline{\mathcal{H}}+K+\|g\|_{C^{1,1}(\Omega)}\right)^{2}
$$

if $h \in\left(0, h_{0}\right]$. In light of (6.15) one can take $\nu=N \mu^{-2}$ for an appropriate $N$ and then

$$
\eta_{\mu}^{4}\left[\left(\Delta_{r} v_{h}(x)\right)^{-}\right]^{2} \leq \max _{y \in Q \backslash Q^{o}} \eta_{\mu}^{4}\left[\left(\Delta_{r} v_{h}(y)\right)^{-}\right]^{2}+N \mu^{-2}\left(\overline{\mathcal{H}}+K+\|g\|_{C^{1,1}(\Omega)}\right)^{2}
$$

for $x \in Q^{o}$. We will only concentrate on $\mu \geq 3 h$, when $\eta_{\mu}=0$ outside $\Omega_{3 h}$. In that case, for any $y \in Q \backslash Q^{o}$, either $y \notin \Omega_{3 h}$ implying that

$$
\eta_{\mu}^{4}\left[\left(\Delta_{r} v_{h}\right)^{-}\right]^{2}(y)=0
$$

or else $y \in \Omega_{3 h} \cap\left(h \Lambda_{\infty}\right)$ but

$$
(\delta / 2)\left|\Delta_{h, r} v_{h}(y)\right| \leq \overline{\mathcal{H}}+K .
$$

It follows that as long as $h \in\left(0, h_{0}\right], x \in Q^{\circ}$, and $\mu \geq 3 h$ we have

$$
\eta_{\mu}^{4}\left[\left(\Delta_{r} v_{h}\right)^{-}(x)\right]^{2} \leq N \mu^{-2}\left(\overline{\mathcal{H}}+K+\|g\|_{C^{1,1}(\Omega)}\right)^{2} .
$$

If $x$ is such that $\rho(x) \geq 6 \lambda h$, take $\mu=\mu_{0} \wedge(\rho(x) /(2 \lambda))$, which is bigger than $3 h$ provided that $h \leq \mu_{0} / 3$. In that case also

$$
\rho(x)=2 \lambda[\rho(x) /(2 \lambda)] \geq 2 \lambda \mu,
$$

so that $\eta_{\mu}(x)=1$ and we conclude from (6.16) that

$$
\begin{gathered}
\rho(x)\left(\Delta_{r} v_{h}\right)^{-}(x) \leq N\left(\overline{\mathcal{H}}+K+\|g\|_{C^{1,1}(\Omega)}\right), \\
(\rho(x)-6 \lambda h)\left(\Delta_{r} v_{h}\right)^{-}(x) \leq N\left(\overline{\mathcal{H}}+K+\|g\|_{C^{1,1}(\Omega)}\right)
\end{gathered}
$$

for $x \in Q^{o}$ such that $\rho(x) \geq 6 \lambda h$. However, the second relation in (6.17) is obvious for $\rho(x) \leq 6 \lambda h$. 
As a result of all the above arguments we see that (6.17) holds in $h \Lambda_{\infty}$ for any $r$ whenever $h \in\left(0, h_{0}\right]$.

Finally, since $P_{h}\left[v_{h}\right] \leq K$ in $\Omega_{h}$ we have that

$$
\begin{aligned}
2 \delta^{-1} \sum_{r}\left(\Delta_{r} v_{h}\right)_{+} \leq & (\delta / 2) \sum_{r}\left(\Delta_{r} v_{h}\right)_{-} \\
& -2 \delta^{-1} \sum_{r \geq 1}\left|\delta_{r} v_{h}\right|+(\delta / 2)\left(v_{h}\right)_{+}-2 \delta^{-1}\left(v_{h}\right)_{-}+K
\end{aligned}
$$

which after being multiplied by $\rho-6 h$ along with (6.17) and Lemma 6.3 leads to (6.14) on $h \Lambda_{\infty}$. As is explained in the beginning of the proof, this finishes proving the lemma.

Proof of Theorem 6.1. Owing to Assumption 6.1 (iii), by Corollary 2.7 of [12], which is applicable in light of Lemmas 6.3 and 6.4, there exists a constant $M$ such that for all sufficiently small $h$ and $x, y \in \mathbb{R}^{d}$ we have

$$
\left|v_{h}(x)-v_{h}(y)\right| \leq M(|x-y|+h) .
$$

Here Assumptions 6.1(iii) plays a crucial role.

After that our theorem is proved in exactly the same way as Theorem 8.7 of [10] on the basis of Lemmas 6.3 and 6.4 and the fact that the derivatives of $v$ are weak limits of finite differences of $v_{h}$ as $h \downarrow 0$ (see the proof of Theorem 8.7 of [10]). One also uses the fact that there are sufficiently many pure second order derivatives in directions of the $l_{i}$ 's to conclude from their boundedness that the Hessian of $v$ is bounded. The theorem is proved.

7. Proof of Theorem 3.2. The functions $\mathcal{H}$ from Section 4 and $\mathcal{P}$ from Section 1 are instances of $\mathcal{H}$ and $\mathcal{P}$ from Section 6 . To see this, of course, one has to change the constant $\delta$ in Section 6 and renumber the $l_{i}$ 's in Section 1. We also take into account that $\hat{\delta} \leq \delta / 4$ which allows us to match (4.1) and (4.2) with the requirements of Assumption 6.1 (i). Furthermore, $\overline{\mathcal{H}}=\bar{H}$. Therefore, Theorem 6.1 is applicable and yields a unique $v \in C^{0,1}(\bar{\Omega}) \cap C_{\text {loc }}^{1,1}(\Omega)$ such that $v=g$ on $\partial \Omega$, estimates $(6.3)$, that is (1.3), hold true, and

$$
\max \left[\mathcal{H}\left(v, D v, v_{\left(l_{1}\right)\left(l_{1}\right)}, \ldots, v_{\left(l_{m}\right)\left(l_{m}\right)}, x\right), \mathcal{P}\left(v, D v, v_{\left(l_{1}\right)\left(l_{1}\right)}, \ldots, v_{\left(l_{m}\right)\left(l_{m}\right)}\right)-K\right]=0
$$

in $\Omega$ (a.s.). In light of the construction of $\mathcal{H}$ and $\mathcal{F}$ in Section 4 this equation coincides with (1.2), so that the only remaining assertions of Theorem 3.2 to prove are that for $p>d$

$$
\|v\|_{W_{p}^{2}(\Omega)} \leq N_{p}\left(\bar{H}+K+\|g\|_{W_{p}^{2}(\Omega)}\right)
$$

and estimate (1.5) holds. The latter follows from other assertions of Theorem 3.2 by Remark 1.3, so that we may concentrate on (7.1).

Observe that

$$
\max (H(u, x), P(u)-K)=P(u)+G(u, x),
$$

where $G(u, x)=(H(u, x)-P(u)+K)_{+}-K$ and, owing to condition $(3.13), G(u, x)=$ $-K$ if

$$
\kappa\left(\sum_{i, j}\left|u_{i j}\right|+\sum_{i}\left|u_{i}\right|\right) \geq \bar{H}+K
$$


If the opposite inequality holds, then

$$
|G(u, x)| \leq|H(u, x)-H(0, x)|+|P(u)|+\bar{H}+K \leq N(\bar{H}+K),
$$

where $N$ depends only on $\delta$ and $d$. It follows that the inequality between the extreme terms in (7.2) holds for all $u$ and $x$. This allows us to apply Theorem 1.2 of [5] and shows that (7.1) holds if $v \in W_{p}^{2}(\Omega)$ or if $w:=v-g \in W_{p}^{2}(\Omega)$. In light of (1.3) it suffices to show that $w \in W_{p}^{2}(D \cap \Omega)$, where $D$ is a neighborhood of $\partial \Omega$.

To prove the latter we use the fact that, according to (3.14), in a neighborhood $D$ of $\partial \Omega$ intersected with $\Omega$ the function $w$ satisfies the equation

$$
\max (\Delta u-u+\Delta g-g, P[u+g]-K)=0,
$$

in which the left-hand side is given by a convex function of $u$ and its derivatives. We may certainly assume that $D \in C^{2}$ and then, by relying on (1.3), find a $\zeta \in C_{0}^{\infty}(\Omega)$ such that $\zeta w=w$ on $\partial(D \cap \Omega)$ and $\zeta w \in W_{p}^{2}(D \cap \Omega)$. Then due to Theorem 1.2 of $[5]$ equation (7.3) with boundary condition $u-\zeta w \in \stackrel{0}{W}_{p}^{2}(D \cap \Omega)$ has a (unique) solution $u \in W_{p}^{2}(D \cap \Omega)$.

By uniqueness of $W_{d, l o c}^{2}(D \cap \Omega) \cap C(\overline{D \cap \Omega})$-solutions we obtain $w=u \in W_{p}^{2}(D \cap \Omega)$ and the theorem is proved.

Acknowledgment. The author is sincerely grateful to Hongjie Dong for pointing out a few glitches in the first version of the paper and to the referees for careful reading resulting in finding quite a bit of misprints.

\section{REFERENCES}

[1] L. A. Caffarelli, Interior a priori estimates for solutions of fully non-linear equations, Ann. Math., 130 (1989), pp. 189-213.

[2] L. A. Caffarelli and X. CABré, Fully nonlinear elliptic equations, American Mathematical Society, Providence, 1995.

[3] X. CABRÉ AND L. A. CAFFARELli, Interior $C^{2, \alpha}$ regularity theory for a class of nonconvex fully nonlinear elliptic equations, J. Math. Pures Appl. (9), 82:5 (2003), pp. 573-612.

[4] M. G. Crandall, M. Kocan, and A. ŚWIȨCH, $L^{p}$-theory for fully nonlinear uniformly parabolic equations, Comm. Partial Differential Equations, 25:11-12 (2000), pp. 1997-2053.

[5] H. Dong, N. V. KRYlov, and X. LI, On fully nonlinear elliptic and parabolic equations in domains with VMO coefficients, Algebra i Analiz, 24:1 (2012), pp. 54-95.

6] D. Gilbarg and N. S. Trudinger, Elliptic Partial Differential Equations of Second Order, Series: Classics in Mathematics, Springer, Berlin, Heidelberg, New York, 2001.

[7] J. Kovats, Differentiability properties of solutions of nondegenerate Isaacs equations, Nonlinear Anal., 71:12 (2009), pp. e2418-e2426.

[8] N. V. Krylov, Nonlinear elliptic and parabolic equations of second order, Nauka, Moscow, 1985 in Russian; English translation Reidel, Dordrecht, 1987.

[9] N. V. Krylov, On Bellman's equations with VMO coefficients, Methods and Applications of Analysis, 17:1 (2010), pp. 105-122.

[10] N. V. KRYLOv, On a representation of fully nonlinear elliptic operators in terms of pure second order derivatives and its applications, Problems of Mathematical Analysis in Russian; English translation: Journal of Mathematical Sciences, New York, 177:1 (2011), pp. 1-26.

[11] N. V. KRYLOV, Interior estimates for second differences of solutions of finite-difference elliptic Bellman's equations, to appear in Math. Comp.

[12] N. V. KRYLOV, Rate of convergence of difference approximations for uniformly nondegenerate elliptic Bellman's equations, preprint.

[13] H.-J. Kuo And N. S. Trudinger, Discrete methods for fully nonlinear elliptic equations. SIAM Journal on Numerical Analysis, 29:1 (1992), pp. 123-135.

[14] N. NAdirashyili ANd S. VlăDUt, Singular viscosity solutions to fully nonlinear elliptic equations, Journal de Mathématiques Pures et Appliquées, 89:2 (2008), pp. 107-113. 
[15] N. S. TRUDINGER, On regularity and existence of viscosity solutions of nonlinear second order, elliptic equations, in Partial differential equations and the calculus of variations, Vol. II, pp. 939-957, Progr. Nonlinear Differential Equations Appl., 2, Birkhäuser Boston, Boston, MA, 1989.

[16] L. WANG, On the regularity theory of fully nonlinear parabolic equations: I, Comm. Pure Appl. Math., 45 (1992), pp. 27-76.

[17] N. WINTER, $W^{2, p}$ and $W^{1, p}$-estimates at the boundary for solutions of fully nonlinear, uniformly elliptic equations, Z. Anal. Anwend., 28:2 (2009), pp. 129-164. 